\title{
The Economic Outcomes of Pharmacist Interventions at Critical Care Services of Private Hospital in Riyadh City, Saudi Arabia
}

\begin{abstract}
Yousef Ahmed Alomi* iD, The Former General Manager of General Administration of Pharmaceutical Care. Former Head, National Clinical Pharmacy and Pharmacy Practice. Former Head, Pharmacy R\&D Administration, Ministry of Health, Riyadh, SAUDI ARABIA.

Manal El-Bahnasawi, Head, Pharmacy Services Sulaiman Al-Habib Medical Group, Riyadh, SAUDI ARABIA.

Alaa Elemam, Clinical Pharmacy Staff, Pharmacy Services, Sulaiman Al-Habib Medical Group, Riyadh, SAUDI ARABIA. Tasneem Shaweesh, Clinical Pharmacy Staff, Pharmacy Services, Sulaiman Al-Habib Medical Group, Riyadh, SAUDI ARABIA.

Edmarie Janine Antonio, Clinical Pharmacy Staff, Pharmacy Services, Sulaiman Al-Habib Medical Group, Riyadh, SAUDI ARABIA.

\section{Correspondence:}

Dr. Yousef Ahmed Alomi, The Former General Manager of General Administration of Pharmaceutical Care Former Head, National Clinical Pharmacy and Pharmacy Practice Former Head, Pharmacy R\&D Administration Ministry of Health, Riyadh, SAUDI ARABIA.
\end{abstract}

Phone no: +966 504417712 E-mail:yalomi@gmail.com

Received: 3-12-2019

Approved: 29-5-2019

Copyright: ๑ the author(s),publisher and licensee Pharmacology, Toxicology and Biomedical Reports. This is an open-access article distributed under the terms of the Creative Commons Attribution NonCommercial License, which permits unrestricted non-commercial use, distribution, and reproduction in any medium, provided the original work is properly cited.

This is an open access article distributed under the terms of the Creative Commons Attribution-NonCommercial-ShareAlike 4.0 License

Access this article online

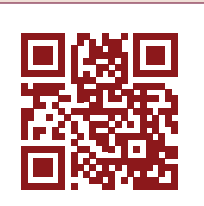

www.ptbreports.org

DOI:

10.5530/PTB.2019.5.34

\begin{abstract}
Objectives: The study aims to explore the economic outcomes of the clinical pharmacist at critical care units at a private hospital in Riyadh city, Saudi Arabia in a 6 month study and prevent additional medication costs. Methods: It was a 6-months cross-sectional study from January to June 2016 in adults critical care unit. It was a thirty-bed critical care bed consisting of trauma, medical, surgical and maternity critical care cases. The pharmacist monitored all patients through daily medical round and documents any pharmacist intervention. The pharmacist intervention system used an international study model, measure level of activity, rational of clinical intervention, recommendation, patient outcome and pharmacoeconomic impact related estimated cost avoidance. Results: The total number of pharmacist interventions were $(1,222)$ provided to $(1,124)$ patients. The complete cost avoidance from pharmacist intervention over the study period was (220,882.10 UD) while over one year was (441,764.2 USD). The highest cost avoidance of critical care interventions was from potentially significant $51.00 \%(102,581.78$ USD) and potentially serious $32.28 \%(99,162.29$ USD). The majority of cost avoidance came from anti-infective agents $(73,408.95$ USD) followed by nutrition and blood agents $(61,182.97$ USD) and cardiovascular medications (27,584.52 USD). The most rational clinical activities cost avoidance was from others type $(102,168.44$ USD) followed by inappropriate dose $(30,504.16$ USD) and TPN consultations (25,313.99 USD). The most patient outcome related to cost avoidance was patient condition improved (120,036.38 USD) followed by unknown outcomes (47,067.85 USD) and laboratory values improved $(21,661.55$ USD). The most pharmacoeconomic cost avoidance impact was a reduction in the cost drug therapy $79.94 \%(163,747.96$ USD) and the patient length of hospital stay decreased $1.15 \%$ $(32,846.49$ USD). Conclusion: The critical care clinical pharmacist prevent the additional cost of drugrelated problems occurrences and economic burden on the healthcare system at a private hospital in the Kingdom of Saudi Arabia. Expanding clinical pharmacist at critical care services is highly recommended to all private hospitals in Saudi Arabia.

Key words: Economic outcomes, Pharmacist, Interventions, Critical care services, Private hopsital, Riyadh, Saudi Arabia.
\end{abstract}

\section{INTRODUCTION}

In the past several years during pharmacy strategic plan at the Ministry of Health (MOH) in Saudi Arabia. ${ }^{1}$ There were several pharmacy practice programs established. ${ }^{2}$ There were several measures tools to follow up the programs and measure clinical outcomes and cost avoidance in pharmacy and healthcare system. ${ }^{1}$ Several previous studies across the world showed the reduction of the economic burden and save cost through pharmacist interventions with an emphasis on critical care services. ${ }^{3-7}$ The pharmacist can prevent medications errors and reduce adverse drug reactions and consequences the cost avoidance., ${ }^{5,6}$ Besides, the current site country conducted several investigations about the economic impact of the pharmacist at clinical services. They showed with drug information services, total parental nutrition services, critical care services, poisoning services. ${ }^{8-11}$ The majority of the studies done at the governmental hospital. It is hard to find a study conducted at private institutions. ${ }^{12}$ The aim of the study is to explore the economic outcome of pharmacist interventions at critical care department at the most prominent private hospital in Riyadh city, Saudi Arabia.

\section{METHODS}

It was a prospective cohort analysis from January to June 2016 in adults critical care unit. It was a thirty-bed critical care unit that consisted of trauma, medical, surgical and maternity critical care cases. Dr. Sulaiman Al-Habib Medical Group (HMG) is one of the largest healthcare providers in the Gulf Cooperation Council (GCC) Countries; currently there are 14 medical which are located in Saudi Arabia (Riyadh and Qassim) and in the UAE (Dubai). HMG is also developing one of the largest private medical cities in Saudi Arabia. In 1995, the core of the Medical Group of Dr. Sulaiman Al Habib was built in Olya area in the Saudi capital, Riyadh, with accordance to an advanced vision that applies the highest international healthcare standards; in providing fully integrated premium health services with a capacity of 241 beds. Furthermore, the complex comprises premium hospitals and clinics of fully integrated maternity hospital, bone, joint and spine surgery hospital, dermatology and plastic surgery centre, ophthalmology and laser/vision correction surgical centre, specialized infertility treatment and reproduction assistance centre. The pharmacies of HMG apply the new technological methods at all work 
phases. HMC Pharmacies have 4 in-patient pharmacy satellites and 3 outpatient pharmacies including the main. Pharmacies work 24/7 with an integrated team of 97 pharmacists with mutiple specialities. The pharmacy's work depends on the patient's medical record system as it helps the pharmacist to provide greater care and protection against the conflict between medicines, especially concerning chronic diseases. The pharmacist monitored all patients through daily medical round and documents any pharmacist intervention. The pharmacist intervention system used an international study model, measure level of activity, rational of clinical intervention, recommendation, patient outcome and pharmacoeconomic impact. The estimated cost avoidance of prevents drug-related problems. ${ }^{13,14}$ The data was gathered and analyzed using the Monkey survey system.

\section{RESULTS}

The total number of pharmacist interventions were $(1,222)$ provided to $(1,124)$ patients. Of those $682(44.19 \%)$ was male, $682(55.81 \%)$ was female, the majority of patients were Saudi 956 (78.23\%) The most patients in aged 65 years and above was $459(37.59 \%)$ followed by age (18-40) was 368 (30.14\%) and age (40-65) 263 (21.54\%) (Table 1). The total cost avoidance from pharmacist intervention over the study period was (220,882.10 UD) while over one year was (441,764.2 USD). The cost avoidance during the study period was from female related was $44.41 \%$ (99,236.14 USD) while male avoidance cost was 55.59\% (121,645.96 USD). Nationality related cost avoidance was $79.27 \%(195,711.07$ USD) of saudi patients while $20.73 \%$ (25,171.03 USD) of non-saudi patients. The highest cost avoidance age was age over 65 years was $37.38 \%$ (90,630.48 USD), followed by age (16-40) was 30.59\% (41,693.28 USD) and age (40-65 years) was $20.46 \%$ (28,607.03 USD) (Table $2-4)$. The most cost avoidance came from senior registrar prescriber $32.09 \%(273,796.51$
Table 1: Patient demographic information.

\begin{tabular}{|c|c|c|}
\hline \multicolumn{3}{|l|}{ Age } \\
\hline Answer Options & Response Count & Response Percent \\
\hline $0-30$ days & 36 & $2.95 \%$ \\
\hline 1 month- 6 years & 56 & $4.59 \%$ \\
\hline $6-12$ years & 17 & $1.39 \%$ \\
\hline $12-18$ years & 22 & $1.80 \%$ \\
\hline $18-40$ years & 368 & $30.14 \%$ \\
\hline $40-65$ years & 263 & $21.54 \%$ \\
\hline 65 or older & 459 & $37.59 \%$ \\
\hline \multicolumn{2}{|l|}{ answered question } & 1221 \\
\hline \multicolumn{2}{|l|}{ skipped question } & 1 \\
\hline \multicolumn{3}{|l|}{ Sex } \\
\hline Answer Options & Response Count & Response Percent \\
\hline Female & 540 & $44.19 \%$ \\
\hline Male & 682 & $55.81 \%$ \\
\hline \multicolumn{2}{|l|}{ answered question } & 1222 \\
\hline \multicolumn{2}{|l|}{ skipped question } & 0 \\
\hline \multicolumn{3}{|l|}{ Nationality } \\
\hline Answer Options & Response Count & Response Percent \\
\hline Saudi & 956 & $78.23 \%$ \\
\hline Non-Saudi & 266 & $21.77 \%$ \\
\hline \multicolumn{2}{|l|}{ answered question } & 1222 \\
\hline \multicolumn{2}{|l|}{ skipped question } & 0 \\
\hline
\end{tabular}

Table 2: Gender VS cost avoidance.

\begin{tabular}{|c|c|c|c|c|c|c|c|c|c|c|}
\hline \multirow{2}{*}{$\begin{array}{l}\text { Sex } \\
\text { No }\end{array}$} & \multirow[b]{2}{*}{ Cost } & \multicolumn{3}{|c|}{ Female } & \multicolumn{3}{|c|}{ Male } & \multicolumn{3}{|c|}{ Total } \\
\hline & & No & $\%$ & $\begin{array}{c}\text { Cost } \\
\text { avoidance }\end{array}$ & No & $\%$ & $\begin{array}{c}\text { Cost } \\
\text { avoidance }\end{array}$ & No & $\%$ & $\begin{array}{c}\text { Cost } \\
\text { avoidance }\end{array}$ \\
\hline 1a & 26.17 & 5 & $62.50 \%$ & 130.85 & 3 & $37.50 \%$ & 78.51 & 8 & $0.00 \%$ & 209.36 \\
\hline $1 b$ & 54.89 & 9 & $28.13 \%$ & 494.01 & 23 & $71.88 \%$ & $1,262.47$ & 32 & $0.00 \%$ & $1,756.48$ \\
\hline $2 a$ & 30.35 & 0 & $0.00 \%$ & 0.00 & 0 & $0.00 \%$ & 0.00 & 0 & $0.00 \%$ & 0.00 \\
\hline $2 \mathrm{~b}$ & 30.35 & 67 & $53.60 \%$ & $2,033.45$ & 58 & $46.40 \%$ & $1,760.30$ & 125 & $0.00 \%$ & $3,793.75$ \\
\hline $2 \mathrm{c}$ & 30.35 & 0 & $0.00 \%$ & 0.00 & 0 & $0.00 \%$ & 0.00 & 0 & $0.00 \%$ & 0.00 \\
\hline $2 \mathrm{~d}$ & 30.35 & 175 & $50.43 \%$ & $5,311.25$ & 172 & $49.57 \%$ & $5,220.20$ & 347 & $1.96 \%$ & $10,531.45$ \\
\hline $2 \mathrm{e}$ & 30.35 & 21 & $46.67 \%$ & 637.35 & 24 & $53.33 \%$ & 728.40 & 45 & $0.00 \%$ & $1,365.75$ \\
\hline $2 \mathrm{f}$ & 34.31 & 2 & $66.67 \%$ & 68.62 & 1 & $33.33 \%$ & 34.31 & 3 & $0.00 \%$ & 102.93 \\
\hline $3 a$ & 260.46 & 159 & $36.72 \%$ & $41,413.14$ & 274 & $63.28 \%$ & $71,366.04$ & 433 & $5.10 \%$ & $112,779.18$ \\
\hline $3 b$ & 109 & 9 & $37.50 \%$ & 981.00 & 15 & $62.50 \%$ & $1,635.00$ & 24 & $4.71 \%$ & $2,616.00$ \\
\hline $3 c$ & 320.7 & 0 & $0.00 \%$ & 0.00 & 0 & $0.00 \%$ & 0.00 & 0 & $2.75 \%$ & 0.00 \\
\hline $4 \mathrm{a}$ & 370.04 & 5 & $83.33 \%$ & $1,850.20$ & 1 & $16.67 \%$ & 370.04 & 6 & $54.90 \%$ & $2,220.24$ \\
\hline $4 \mathrm{~b}$ & 581.75 & 0 & $0.00 \%$ & 0.00 & 0 & $0.00 \%$ & 0.00 & 0 & $1.57 \%$ & 0.00 \\
\hline $4 c$ & $3,562.79$ & 13 & $54.17 \%$ & $46,316.27$ & 11 & $45.83 \%$ & $39,190.69$ & 24 & $5.88 \%$ & $85,506.96$ \\
\hline $4 \mathrm{~d}$ & $6,437.13$ & 0 & $0.00 \%$ & 0.00 & 0 & $0.00 \%$ & 0.00 & 0 & $6.67 \%$ & 0.00 \\
\hline $5 \mathrm{a}$ & $6,592.03$ & 0 & $0.00 \%$ & 0.00 & 0 & $0.00 \%$ & 0.00 & 0 & $7.45 \%$ & 0.00 \\
\hline $5 b$ & $9,466.36$ & 0 & $0.00 \%$ & 0.00 & 0 & $0.00 \%$ & 0.00 & 0 & $7.06 \%$ & 0.00 \\
\hline $5 c$ & $11,837.51$ & 0 & $0.00 \%$ & 0.00 & 0 & $0.00 \%$ & 0.00 & 0 & $1.96 \%$ & 0.00 \\
\hline 6 & $100,000.00$ & 0 & $0.00 \%$ & 0.00 & 0 & $0.00 \%$ & 0.00 & 0 & $0.00 \%$ & 0.00 \\
\hline Total & & 465 & $44.41 \%$ & $99,236.14$ & 582 & $55.59 \%$ & $121,645.96$ & 1047 & $100.00 \%$ & $220,882 \cdot 10$ \\
\hline
\end{tabular}


Table 3: Nationalities VS cost avoidance.

\begin{tabular}{|c|c|c|c|c|c|c|c|c|c|c|}
\hline \multirow{2}{*}{$\begin{array}{l}\text { Sex } \\
\text { No }\end{array}$} & \multirow[b]{2}{*}{ Cost } & \multicolumn{3}{|c|}{ Saudi } & \multicolumn{3}{|c|}{ Non-Saudi } & \multicolumn{3}{|c|}{ Total } \\
\hline & & No & $\%$ & $\begin{array}{c}\text { Cost } \\
\text { avoidance }\end{array}$ & No & $\%$ & $\begin{array}{c}\text { Cost } \\
\text { avoidance }\end{array}$ & No & $\%$ & $\begin{array}{c}\text { Cost } \\
\text { avoidance }\end{array}$ \\
\hline la & 26.17 & 7 & $87.50 \%$ & 183.19 & 1 & $12.50 \%$ & 26.17 & 8 & $0.00 \%$ & 209.36 \\
\hline $1 b$ & 54.89 & 30 & $93.75 \%$ & $1,646.70$ & 2 & $6.25 \%$ & 109.78 & 32 & $0.00 \%$ & $1,756.48$ \\
\hline $2 \mathrm{a}$ & 30.35 & 0 & $0.00 \%$ & 0.00 & 0 & $0.00 \%$ & 0.00 & 0 & $0.00 \%$ & 0.00 \\
\hline $2 b$ & 30.35 & 85 & $68.00 \%$ & $2,579.75$ & 40 & $32.00 \%$ & $1,214.00$ & 125 & $0.00 \%$ & $3,793.75$ \\
\hline $2 c$ & 30.35 & 0 & $0.00 \%$ & 0.00 & 0 & $0.00 \%$ & 0.00 & 0 & $0.00 \%$ & 0.00 \\
\hline $2 \mathrm{~d}$ & 30.35 & 240 & $69.16 \%$ & $7,284.00$ & 107 & $30.84 \%$ & $3,247.45$ & 347 & $1.96 \%$ & $10,531.45$ \\
\hline $2 \mathrm{e}$ & 30.35 & 34 & $75.56 \%$ & $1,031.90$ & 11 & $24.44 \%$ & 333.85 & 45 & $0.00 \%$ & $1,365.75$ \\
\hline $2 \mathrm{f}$ & 34.31 & 3 & $100.00 \%$ & 102.93 & 0 & $0.00 \%$ & 0.00 & 3 & $0.00 \%$ & 102.93 \\
\hline $3 a$ & 260.46 & 387 & $89.38 \%$ & $100,798.02$ & 46 & $10.62 \%$ & $11,981.16$ & 433 & $5.10 \%$ & $112,779.18$ \\
\hline $3 b$ & 109 & 17 & $70.83 \%$ & $1,853.00$ & 7 & $29.17 \%$ & 763.00 & 24 & $4.71 \%$ & $2,616.00$ \\
\hline $3 c$ & 320.7 & 0 & $0.00 \%$ & 0.00 & 0 & $0.00 \%$ & 0.00 & 0 & $2.75 \%$ & 0.00 \\
\hline $4 a$ & 370.04 & 5 & $83.33 \%$ & $1,850.20$ & 1 & $16.67 \%$ & 370.04 & 6 & $54.90 \%$ & $2,220.24$ \\
\hline $4 \mathrm{~b}$ & 581.75 & 0 & $0.00 \%$ & 0.00 & 0 & $0.00 \%$ & 0.00 & 0 & $1.57 \%$ & 0.00 \\
\hline $4 c$ & $3,562.79$ & 22 & $91.67 \%$ & $78,381.38$ & 2 & $8.33 \%$ & $7,125.58$ & 24 & $5.88 \%$ & $85,506.96$ \\
\hline $4 \mathrm{~d}$ & $6,437.13$ & 0 & $0.00 \%$ & 0.00 & 0 & $0.00 \%$ & 0.00 & 0 & $6.67 \%$ & 0.00 \\
\hline $5 a$ & $6,592.03$ & 0 & $0.00 \%$ & 0.00 & 0 & $0.00 \%$ & 0.00 & 0 & $7.45 \%$ & 0.00 \\
\hline $5 b$ & $9,466.36$ & 0 & $0.00 \%$ & 0.00 & 0 & $0.00 \%$ & 0.00 & 0 & $7.06 \%$ & 0.00 \\
\hline $5 c$ & $11,837.51$ & 0 & $0.00 \%$ & 0.00 & 0 & $0.00 \%$ & 0.00 & 0 & $1.96 \%$ & 0.00 \\
\hline 6 & $100,000.00$ & 0 & $0.00 \%$ & 0.00 & 0 & $0.00 \%$ & 0.00 & 0 & $0.00 \%$ & 0.00 \\
\hline Total & & 830 & $79.27 \%$ & $195,711.07$ & 217 & $20.73 \%$ & $25,171.03$ & 255 & $100.00 \%$ & $220,882 \cdot 10$ \\
\hline
\end{tabular}

USD) and consultant prescriber 63.61\% (180,463.62USD). The highest cost avoidance of critical care interventions was from potentially significant $51.00 \%$ (102,581.78 USD) and potentially serious $32.28 \%(99,162.29$ USD) (Table 5 and 6). The majority of cost avoidance came from antiinfective agents $(73,408.95$ USD) followed by nutrition and blood agents (61,182.97 USD) and cardiovascular medications (27,584.52 USD) (Table 7). The most rational clinical activities cost avoidance was from others type (102,168.44 USD) followed by inappropriate dose $(30,504.16$ USD) and TPN consultations (25,313.99 USD). While the most pharmacist recommendations type related to cost avoidance was change dose (65,599.18 USD) followed by other recommendations $(43,624.15$ USD) and the drug discontinued (37,896.28 USD) (Table 8 and 9). The most patient outcome related to cost avoidance was patient condition improved (120,036.38 USD) followed by unknown outcomes $(47,067.85$ USD) and laboratory values improved (21,661.55 USD). The most Pharmacoeconomic cost avoidance impact was a reduction in the cost of drug therapy $79.94 \%$ (163,747.96 USD) and the patient length of hospital stay decreased $1.15 \%(32,846.49$ USD) (Table 10 and 11). The most type cost avoidance of intervention used was type [3a-additional treatment - 260.46\$ 433 (41.36\%) followed by type [2d - dosage adjustments (no potential for harm - 30.35\$] 347 (33.14\%) and type (2b - therapeutic duplication - 30.35\$) 125 (11.94\%) (Table 12).

\section{DISCUSSION}

Many pharmacy practice programs founded during Pharmacy strategic plan. ${ }^{1,15}$ That has included drug information services, medication safety program, pain management program, stewardship antimicrobial program and critical care program programs. ${ }^{16-19}$ All those programs need to measure the impact and validate the cost requirement. The pharmacy administration at the ministry of health released key performance indicators to follow up and measure the clinical and economic impact of the program. The pharmacy strategic plan started implementation at private hospitals. Authors working at the most prominent private hospital in Riyadh city wish to measure the economic outcome of critical care pharmacy services. The findings should very high cost avoidance as the impact of pharmacist intervention at critical care services. That almost similar what report by Aljbouri $\mathrm{TM}^{12}$ and MacLaren, $\mathrm{R}$ et al. in reduction infection-related problems, ${ }^{3}$ but higher than what reported by Schumock GT et al. that's related newly established of clinical pharmacy services at current site and the different times between two studies with incremental cost. ${ }^{7}$ However, it is lower cost avoidance what reported by MacLaren $\mathrm{R}$ et al. with cardiologic diseases. ${ }^{4}$ There are not any differences in the cost avoidance among gender because both of them received the same service without differences in the numbers. The majority of cost avoidance related to Saudi patient because most of the patients were Saudi. The findings showed high-cost avoidance from elderly patients that's would be excepted because the geriatrics had several diseases and needed medications and may be exposed to several mistakes and demand for close monitoring for prevention of them. Most of the cost avoidance as results from senior registrar prescriber more than consultant one. That is related to lower level of credential need more education and training related drug therapy. The highest cost avoidance of medications was from anti-infective agent, nutrition product and cardiovascular medications. 


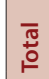

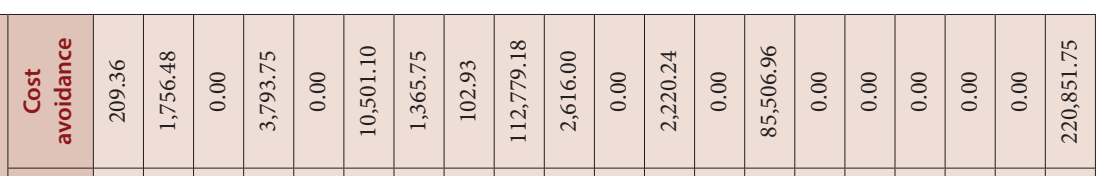

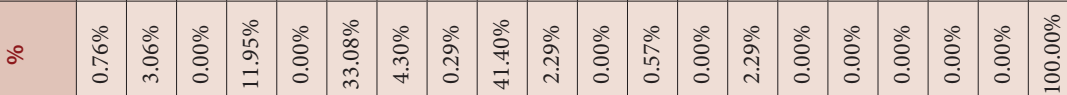

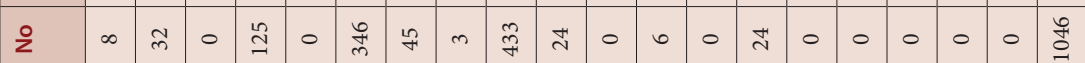

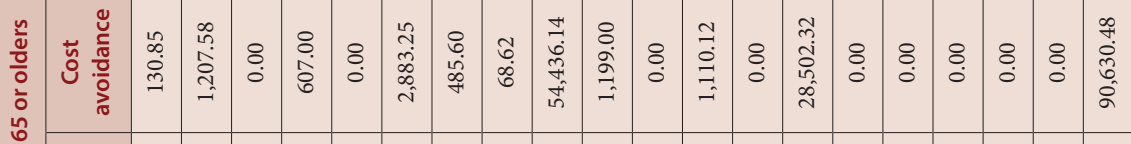

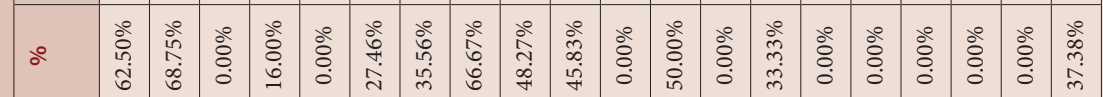

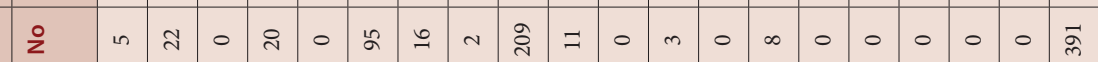

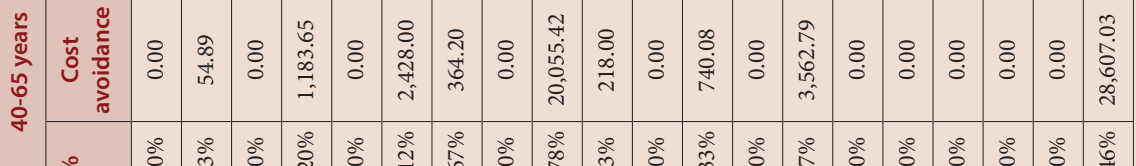

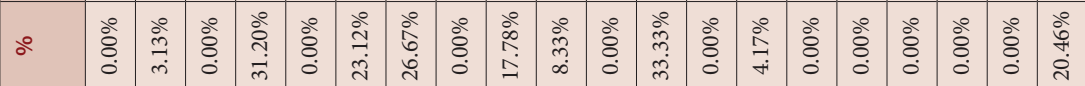

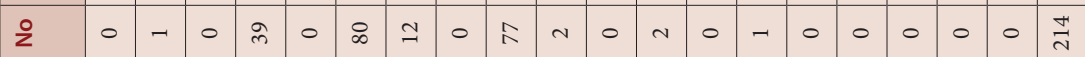

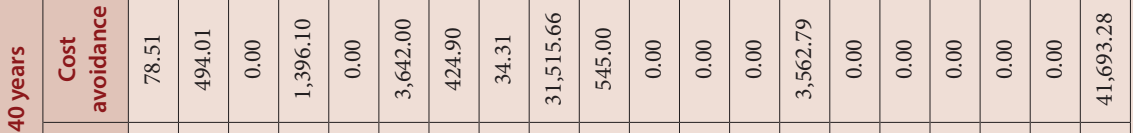

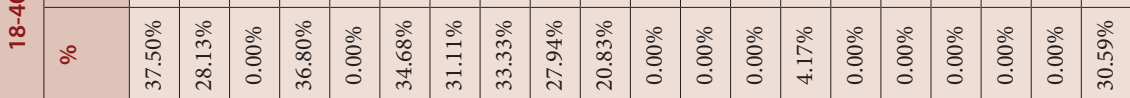

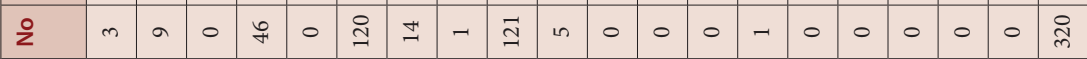

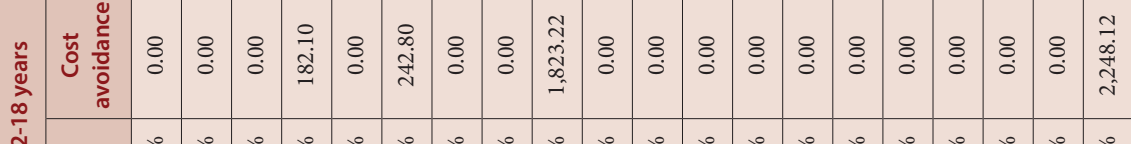

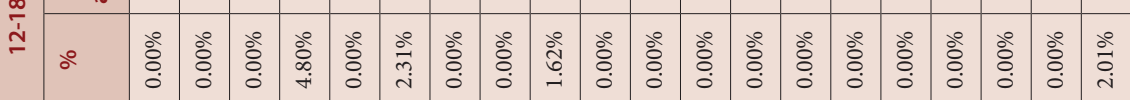
$\begin{array}{llllllllllllllllllllll}\dot{\gamma} & 0 & 0 & 0 & 0 & 0 & 0 & 0 & 0 & n & 0 & 0 & 0 & 0 & 0 & 0 & 0 & 0 & 0 & 0 & \overrightarrow{4}\end{array}$

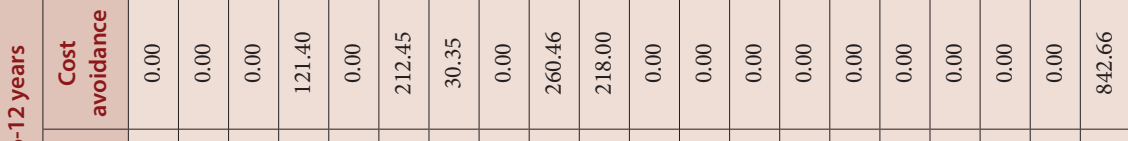
ঢ́ $\begin{array}{llllllllllllllllllllllll}z & 0 & 0 & 0 & + & 0 & n & - & 0 & - & 4 & 0 & 0 & 0 & 0 & 0 & 0 & 0 & 0 & 0 & h\end{array}$

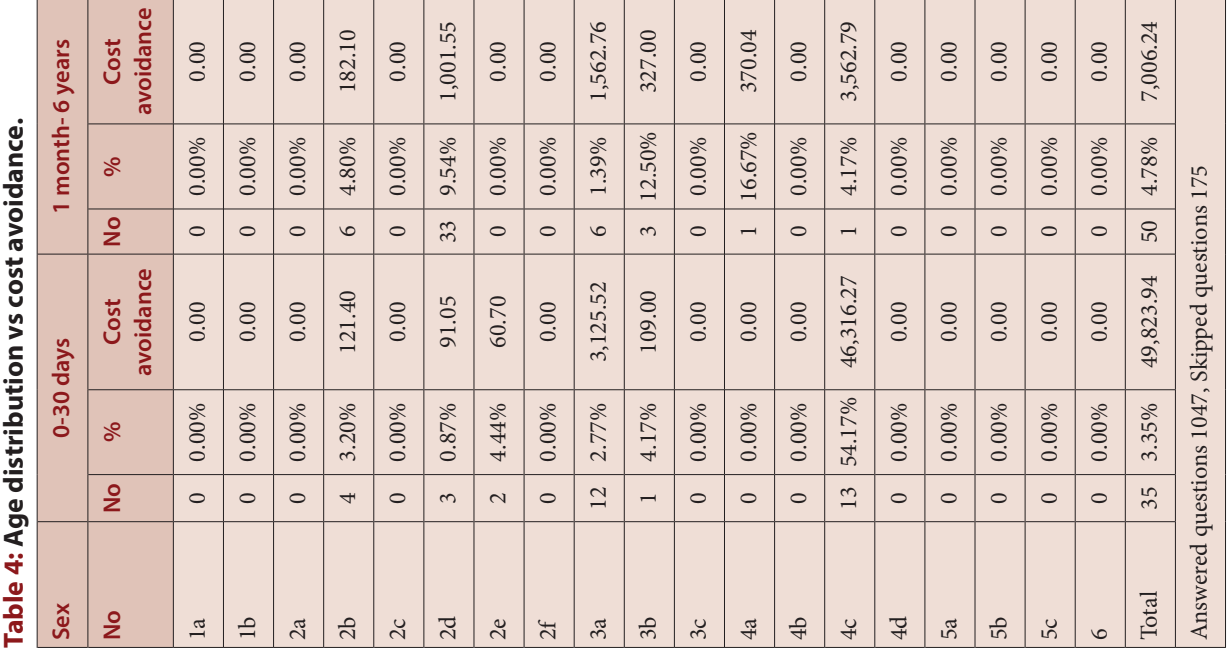




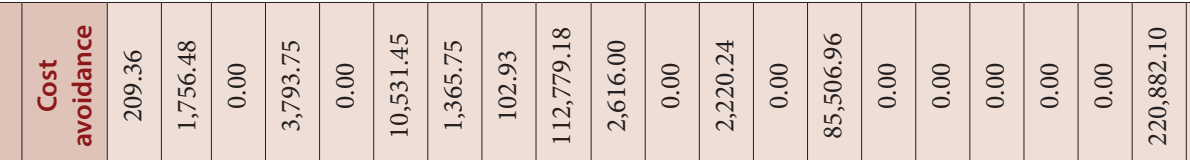

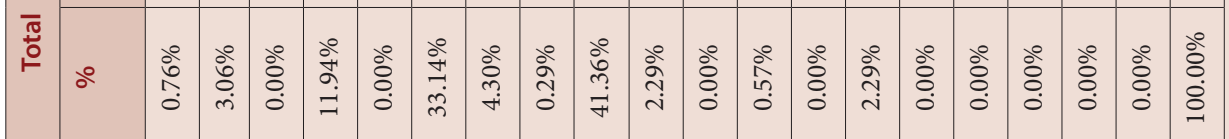

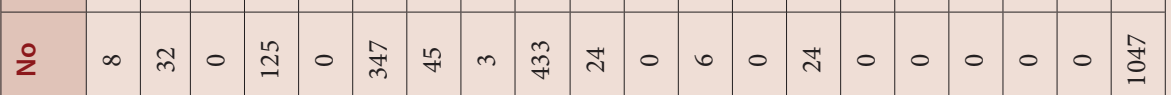

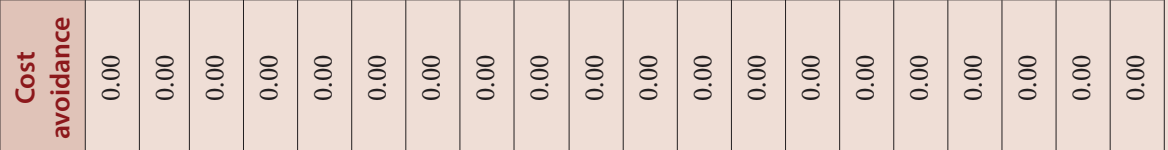

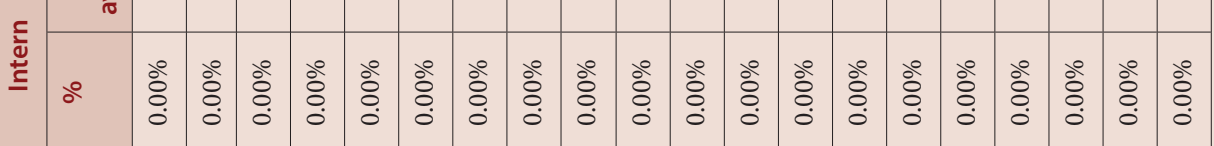
\begin{tabular}{lllllllllllllllllllllllllllll}
\hline & 0 & 0 & 0 & 0 & 0 & 0 & 0 & 0 & 0 & 0 & 0 & 0 & 0 & 0 & 0 & 0 & 0 & 0 & 0 & 0 \\
\hline
\end{tabular}

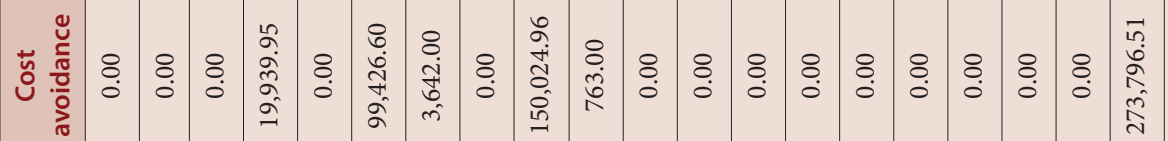

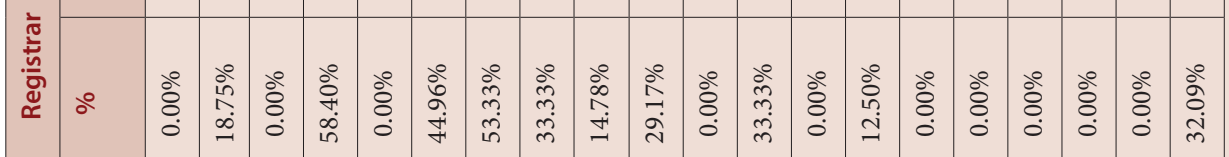

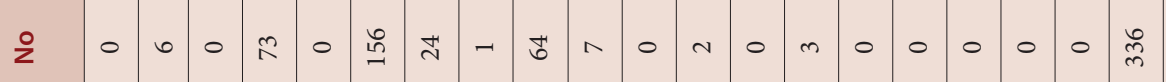

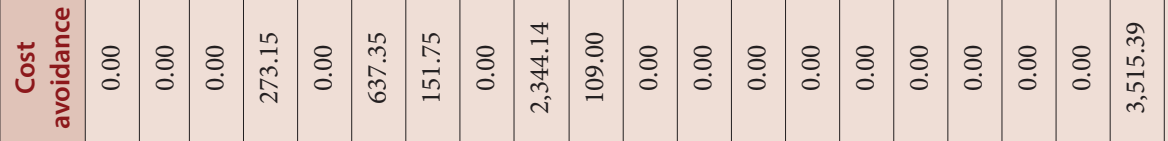

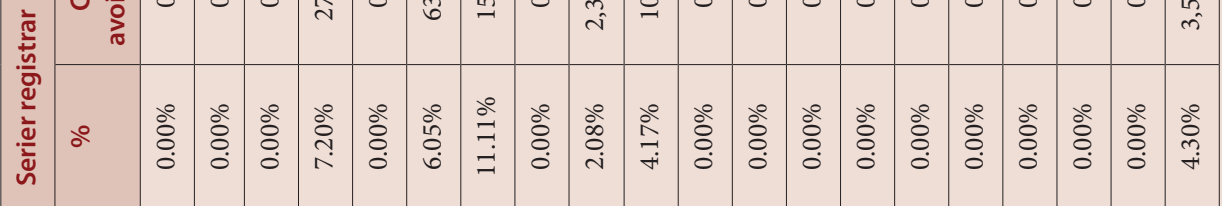
\begin{tabular}{lllllllllllll|llllllllllllll}
\hline & 0 & 0 & 0 & $a$ & 0 & $\overrightarrow{4}$ & $\ln$ & 0 & $a$ & - & 0 & 0 & 0 & 0 & 0 & 0 & 0 & 0 & 0 & 4 \\
\hline
\end{tabular}

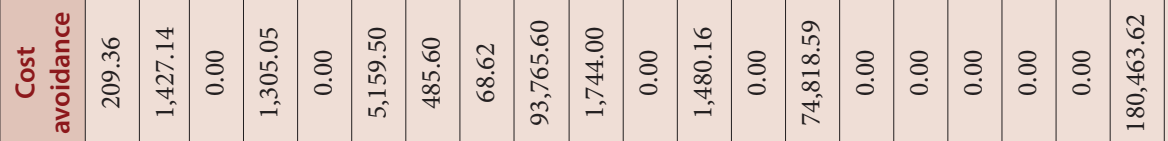

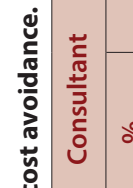

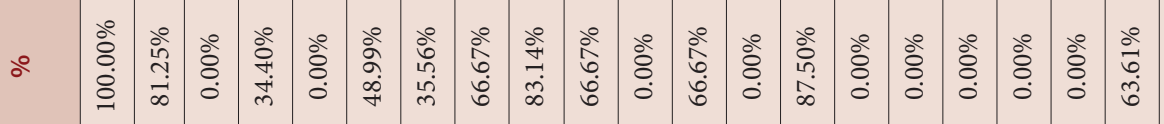

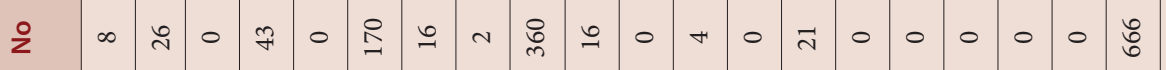

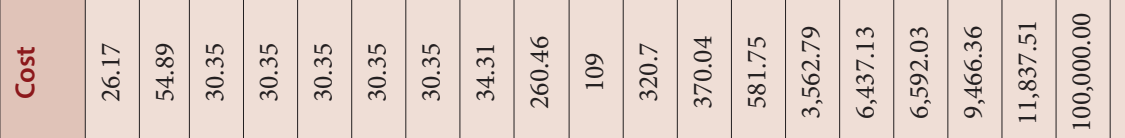




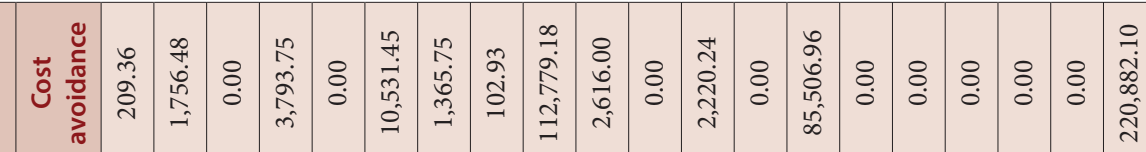

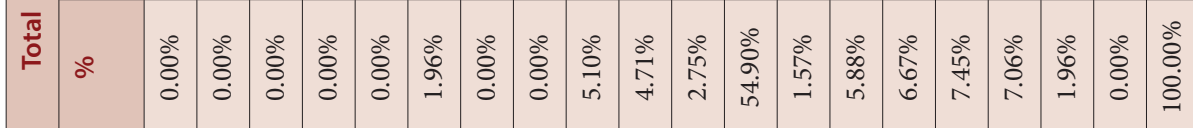

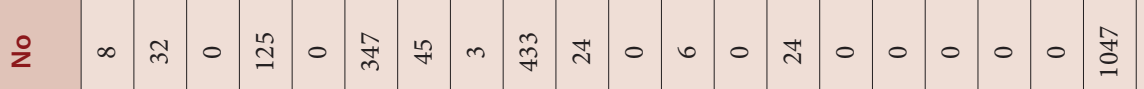

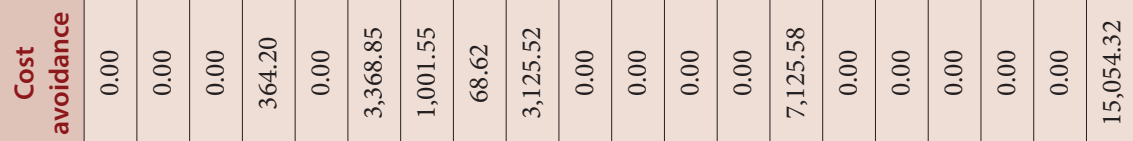

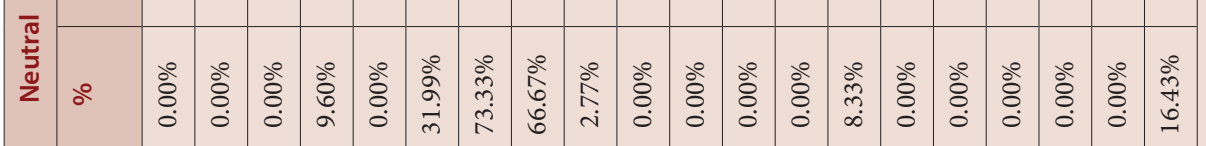

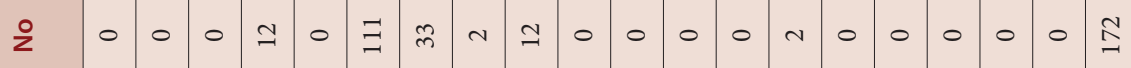

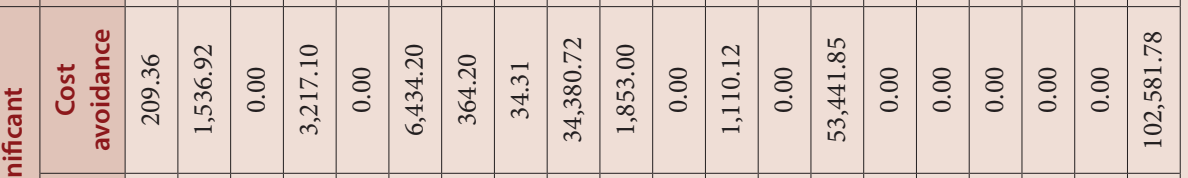

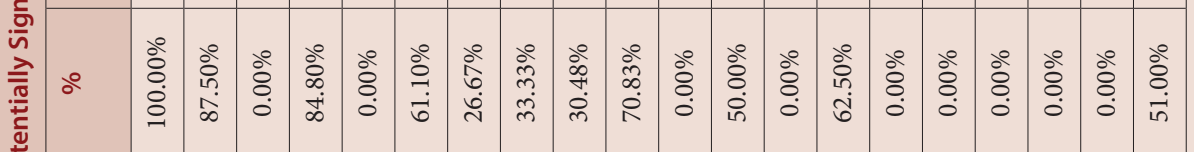
$\stackrel{\circ}{\circ}$

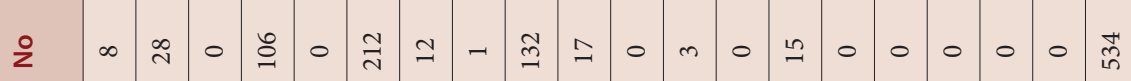

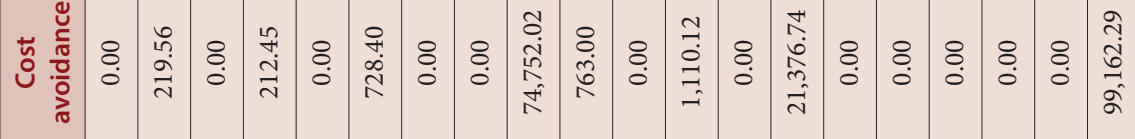

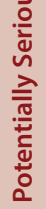

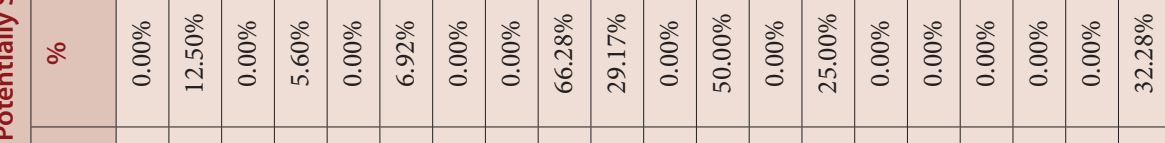

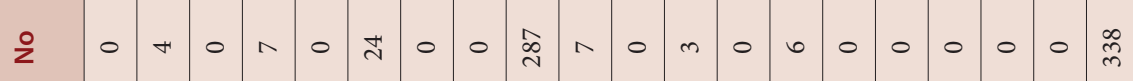

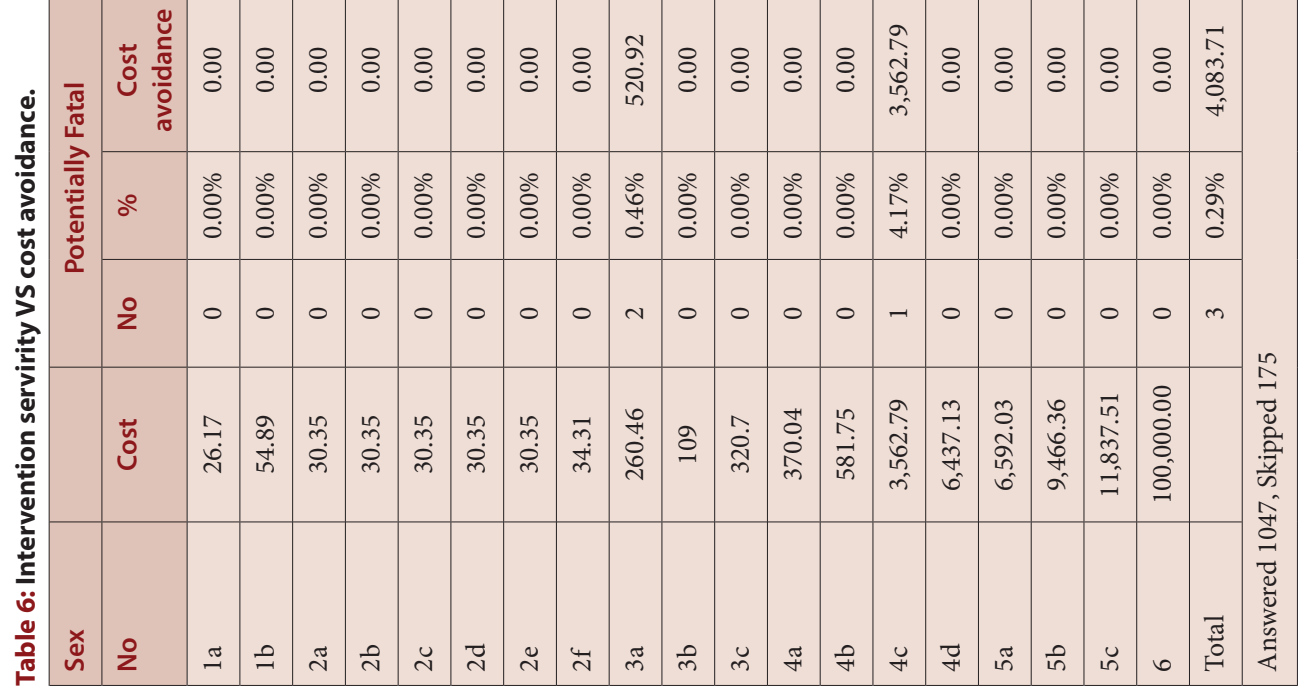




\begin{tabular}{|c|c|c|c|c|c|c|c|c|c|c|c|c|c|c|c|c|c|c|c|c|}
\hline jełol & 官 & 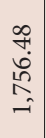 & 0 & 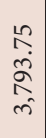 & 0 & 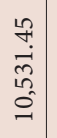 & $\begin{array}{c}n \\
\hat{n} \\
10 \\
0 \\
-1 \\
-1\end{array}$ & 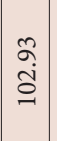 & 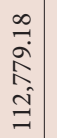 & $\begin{array}{c}0 \\
0 \\
0 \\
0 \\
0 \\
i v\end{array}$ & 0 & 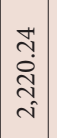 & 0 & 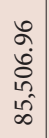 & 0 & 0 & 0 & 0 & 0 & 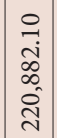 \\
\hline АәчҰО & $\begin{array}{l}n \\
\infty \\
0 \\
\infty \\
-1\end{array}$ & $\begin{array}{l}\hat{i n} \\
\stackrel{n}{n}\end{array}$ & 0 & $\begin{array}{l}\stackrel{0}{1} \\
\stackrel{0}{0} \\
\text { r) }\end{array}$ & 0 & 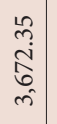 & 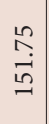 & 0 & $\begin{array}{l}0 \\
+ \\
\infty \\
\not \\
0 \\
0\end{array}$ & 0 & 0 & $\begin{array}{c}0 \\
0 \\
\dot{0} \\
0 \\
m\end{array}$ & 0 & 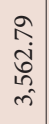 & 0 & 0 & 0 & 0 & 0 & 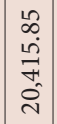 \\
\hline е!รәчұsәеи & 0 & 0 & 0 & $\hat{8}$ & 0 & $\stackrel{+}{\stackrel{H}{I}}$ & 0 & 0 & 0 & 0 & 0 & 0 & 0 & 0 & 0 & 0 & 0 & 0 & 0 & $\vec{c}$ \\
\hline sıәрлоs!p u!YS & 0 & 0 & 0 & 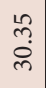 & 0 & 0 & 0 & 0 & $\stackrel{\substack{\infty \\
\infty}}{\infty}$ & 0 & 0 & 0 & 0 & 0 & 0 & 0 & 0 & 0 & 0 & 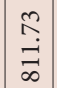 \\
\hline $\begin{array}{l}\text { xuKıеydoıo } \\
\text { pue әson'se }\end{array}$ & 0 & 0 & 0 & $\stackrel{2}{0}$ & 0 & है & 0 & 0 & 0 & 0 & 0 & 0 & 0 & 0 & 0 & 0 & 0 & 0 & 0 & $\begin{array}{c}\vec{i} \\
\infty \\
-\rightarrow\end{array}$ \\
\hline ләрлоь!р әКヨ & 0 & 0 & 0 & 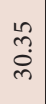 & 0 & $\begin{array}{l}m \\
m \\
\dot{m}\end{array}$ & 0 & 0 & $\begin{array}{l}\overrightarrow{+} \\
\dot{+} \\
\overrightarrow{+} \\
-i \\
-i\end{array}$ & 0 & 0 & 0 & 0 & 0 & 0 & 0 & 0 & 0 & 0 & 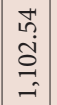 \\
\hline $\begin{array}{c}\text { səseəs!̣ ұu!o! } \\
\text { pue ןеłə|əyso|nวsnw }\end{array}$ & 0 & 0 & 0 & ڤn & 0 & $\hat{8}$ & 0 & 0 & $\begin{array}{l}0 \\
\stackrel{1}{1} \\
0 \\
0\end{array}$ & 0 & 0 & 0 & 0 & 0 & 0 & 0 & 0 & 0 & 0 & $\vec{n}$ \\
\hline poolq pue uo!!!ฺฺnN & $\vec{i}$ & $\begin{array}{l}\hat{\sigma} \\
\stackrel{\sigma}{+}\end{array}$ & 0 & $\begin{array}{l}\infty \\
\stackrel{\mathbb{J}}{\mathrm{N}}\end{array}$ & 0 & $\begin{array}{l}0 \\
0 \\
1 \\
\infty \\
\infty \\
+1\end{array}$ & $\hat{8}$ & 0 & 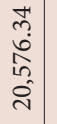 & $\begin{array}{l}0 \\
\stackrel{2}{7}\end{array}$ & 0 & 0 & 0 & $\begin{array}{l}\hat{\sigma} \\
\dot{0} \\
\stackrel{-}{2} \\
\hat{m}\end{array}$ & 0 & 0 & 0 & 0 & 0 & $\mid \begin{array}{c}\hat{\sigma} \\
\dot{j} \\
0 \\
-\hat{\sigma} \\
0\end{array}$ \\
\hline uo!̣ssəגddnsounum! & 0 & 0 & 0 & 0 & 0 & $\begin{array}{l}\stackrel{n}{m} \\
\tilde{m}\end{array}$ & 0 & 0 & 0 & 0 & 0 & 0 & 0 & 0 & 0 & 0 & 0 & 0 & 0 & $\begin{array}{l}n \\
n \\
0 \\
0\end{array}$ \\
\hline ıәрıов!р ұวеגұ-Кıеu!ıก & 0 & 0 & 0 & 0 & 0 & $\hat{8}$ & 0 & 0 & $\begin{array}{c}\infty \\
0 \\
\infty \\
\infty \\
0 \\
i\end{array}$ & 0 & 0 & 0 & 0 & 0 & 0 & 0 & 0 & 0 & 0 & 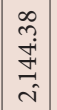 \\
\hline גәрıоs!р КБојоэәеиКפ & 0 & 0 & 0 & $\mid \begin{array}{c}n \\
m \\
\dot{m} \\
\dot{m}\end{array}$ & 0 & $\hat{8}$ & 0 & 0 & $\begin{array}{l}\text { สू. } \\
\text { กิ }\end{array}$ & 0 & 0 & 0 & 0 & 0 & 0 & 0 & 0 & 0 & 0 & $\frac{\hat{\sigma}}{\vec{\sigma}}$ \\
\hline 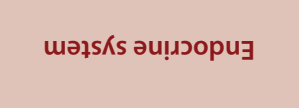 & 0 & 0 & 0 & $\begin{array}{l}n \\
\tilde{n} \\
\tilde{m}\end{array}$ & 0 & $\begin{array}{l}\infty \\
\infty \\
m \\
m \\
m\end{array}$ & $\stackrel{+}{\stackrel{ \pm}{I}}$ & 0 & $\begin{array}{l}\infty \\
2 \\
i \infty \\
\infty \\
m \\
m\end{array}$ & 胞 & 0 & 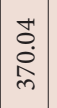 & 0 & 0 & 0 & 0 & 0 & 0 & 0 & $\begin{array}{l}\hat{1} \\
\hat{\sigma} \\
\hat{b} \\
0 \\
i n\end{array}$ \\
\hline 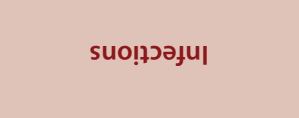 & in & \begin{tabular}{l}
$\infty$ \\
$\infty$ \\
$\infty$ \\
$\infty$ \\
\hdashline
\end{tabular} & 0 & $\vec{\infty}$ & 0 & 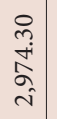 & $\hat{8}$ & $\begin{array}{c}\mathfrak{\alpha} \\
\tilde{o} \\
-\end{array}$ & $\begin{array}{l}H \\
n \\
\infty \\
\infty \\
\infty \\
\infty \\
\infty \\
m\end{array}$ & $\begin{array}{l}8 \\
\dot{0} \\
8 \\
0 \\
-1\end{array}$ & 0 & $\begin{array}{l}0 \\
0 \\
0 \\
0\end{array}$ & 0 & 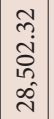 & 0 & 0 & 0 & 0 & 0 & $\begin{array}{l}n \\
\alpha \\
\infty \\
o \\
\vdots \\
0 \\
n \\
n\end{array}$ \\
\hline 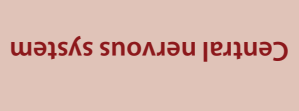 & 0 & 0 & 0 & 0 & 0 & $\begin{array}{l}\stackrel{2}{n} \\
13 \\
\stackrel{n}{7}\end{array}$ & $\begin{array}{l}n \\
0 \\
\dot{a}\end{array}$ & 0 & $\begin{array}{l}8 \\
0 \\
+ \\
0 \\
0 \\
i\end{array}$ & o & 0 & 0 & 0 & 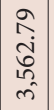 & 0 & 0 & 0 & 0 & 0 & $\left|\begin{array}{c}0 \\
0 \\
\tilde{I} \\
0 \\
0 \\
0\end{array}\right|$ \\
\hline məłsКs Кıоłеג!dsəy & 0 & 0 & 0 & $\begin{array}{l}n \\
\stackrel{2}{a} \\
\dot{a}\end{array}$ & 0 & $\stackrel{n}{\stackrel{n}{+}}$ & 0 & 0 & $\begin{array}{l}\infty \\
0 \\
\stackrel{0}{0} \\
\infty \\
0 \\
\sim\end{array}$ & 0 & 0 & 0 & 0 & 0 & 0 & 0 & 0 & 0 & 0 & $\begin{array}{l}\infty \\
i \\
i n \\
0 \\
1 \\
n\end{array}$ \\
\hline mәłsאs גеןnวse^o!pגeכ & 0 & $\begin{array}{l}\infty \\
\stackrel{2}{a} \\
\stackrel{-}{-}\end{array}$ & 0 & $\begin{array}{l}\stackrel{?}{+} \\
\stackrel{N}{N}\end{array}$ & 0 & 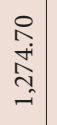 & 0 & 0 & $\begin{array}{l}\mathfrak{N} \\
\hat{i} \\
\stackrel{-}{v} \\
\vec{v}\end{array}$ & $\widehat{\approx}$ & 0 & $\begin{array}{l}\infty \\
0 \\
0 \\
\stackrel{+}{1} \\
1\end{array}$ & 0 & 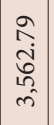 & 0 & 0 & 0 & 0 & 0 & 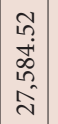 \\
\hline $\begin{array}{c}\text { məłsks } \\
\text { ןeu!|səłu!-oגłseפ }\end{array}$ & 0 & $\begin{array}{l}\infty \\
\stackrel{2}{\circ} \\
\stackrel{0}{\sigma}\end{array}$ & 0 & $\mid \begin{array}{l}0 \\
2 \\
1 \\
\infty \\
\infty \\
+1\end{array}$ & o & $\underset{\infty}{\infty}$ & $\begin{array}{l}10 \\
0 \\
0 \\
\infty \\
\infty\end{array}$ & 0 & $\begin{array}{l}\vec{b} \\
\text { ஸे } \\
0 \\
\infty \\
\infty\end{array}$ & ఏ & 0 & $\begin{array}{l}0 \\
0 \\
0 \\
0 \\
m\end{array}$ & 0 & $\begin{array}{c}\infty \\
i n \\
i n \\
\\
\end{array}$ & 0 & 0 & 0 & 0 & 0 & 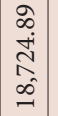 \\
\hline$\stackrel{\times}{\varpi}$ & $\stackrel{\pi}{二}$ & $=$ & กี & ते & $\breve{N}$ & $\vec{\lambda}$ & $\tilde{\sim}$ & 岃 & ñ & ले & $\breve{n}$ & F & fㅇ & U & 军 & กี & กิ & $\ddot{n}$ & 0 & 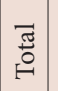 \\
\hline
\end{tabular}




\begin{tabular}{|c|c|c|c|c|c|c|c|c|c|c|c|c|c|c|c|c|c|c|c|c|c|}
\hline |еłOI & $\begin{array}{l}\stackrel{2}{m} \\
\stackrel{i}{े}\end{array}$ & 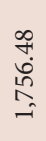 & 0 & 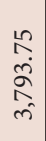 & 0 & $\begin{array}{c}\stackrel{2}{2} \\
\dot{2} \\
10 \\
0 \\
0 \\
-\end{array}$ & $\begin{array}{l}\stackrel{10}{N} \\
10 \\
0 \\
\stackrel{2}{-}\end{array}$ & 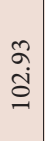 & $\begin{array}{l}\infty \\
\mathfrak{a} \\
\hat{\mathfrak{a}} \\
\hat{\mathfrak{I}} \\
\mathfrak{\Xi}\end{array}$ & $\begin{array}{c}8 \\
0 \\
0 \\
0 \\
0 \\
i\end{array} \mid$ & 0 & 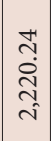 & 0 & \begin{tabular}{l}
\multirow{2}{0}{} \\
రे \\
10 \\
10 \\
$\infty$
\end{tabular} & 0 & 0 & 0 & 0 & 0 & 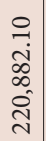 & \\
\hline ләчұО & 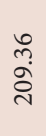 & 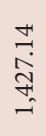 & 0 & 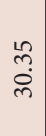 & $\circ$ & 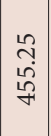 & $\begin{array}{l}\stackrel{10}{2} \\
\stackrel{2}{\sim}\end{array}$ & 0 & $\begin{array}{c}8 \\
0 \\
10 \\
= \\
10 \\
0\end{array}$ & $\begin{array}{c}8 \\
\dot{\infty} \\
\hat{n} \\
\infty \\
-1 \\
-1\end{array}$ & 0 & $\begin{array}{l}\infty \\
\stackrel{0}{0} \\
\stackrel{+}{1} \\
1\end{array}$ & 0 & $\begin{array}{l}= \\
= \\
0 \\
0 \\
\hat{n} \\
m\end{array}$ & 0 & 0 & 0 & 0 & 0 & $\begin{array}{l}\mathbb{Z} \\
\infty \\
0 \\
0 \\
\hat{\sigma} \\
0\end{array}$ & \\
\hline uo!ฺełןnsuoכ $\mathrm{Nd \perp}$ & 0 & 0 & 0 & 0 & 0 & $\mid \begin{array}{c}n \\
m \\
\tilde{m} \\
m\end{array}$ & 0 & 0 & 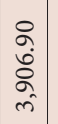 & 0 & 0 & 0 & 0 & 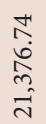 & 0 & 0 & 0 & 0 & 0 & $\begin{array}{l}\stackrel{a}{ } \\
\stackrel{m}{m} \\
\stackrel{m}{n}\end{array}$ & \\
\hline 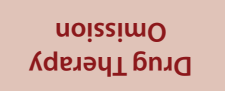 & 0 & 0 & 0 & 0 & 0 & 0 & 0 & 0 & $\begin{array}{l}0 \\
0 \\
1 \\
0 \\
0 \\
1\end{array}$ & 0 & 0 & 0 & 0 & 0 & 0 & 0 & 0 & 0 & 0 & 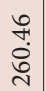 & \\
\hline 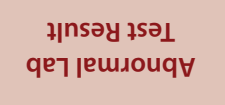 & 0 & 0 & 0 & 0 & 0 & 0 & 0 & 0 & 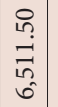 & $\stackrel{\infty}{\vec{\sim}}$ & 0 & 0 & 0 & 0 & 0 & 0 & 0 & 0 & 0 & 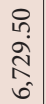 & \\
\hline 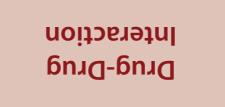 & 0 & 0 & 0 & 0 & 0 & 0 & 0 & 0 & 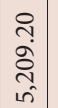 & $\stackrel{\infty}{\vec{\sim}}$ & 0 & $\begin{array}{l}\infty \\
0 \\
\dot{+} \\
\\
\end{array}$ & 0 & 0 & 0 & 0 & 0 & 0 & 0 & 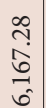 & \\
\hline Kł!!!q!’eduosu| & 0 & 0 & 0 & 0 & 0 & 0 & 0 & 0 & 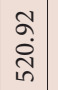 & 0 & 0 & 0 & 0 & 0 & 0 & 0 & 0 & 0 & 0 & 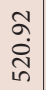 & \\
\hline $\begin{array}{c}\text { ə|nрәчગ્ડ } \\
\text { әرе!ıdoxddeu| }\end{array}$ & 0 & $\begin{array}{l}\infty \\
\text { î } \\
\text { in }\end{array}$ & 0 & 0 & 0 & 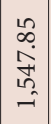 & 0 & 0 & 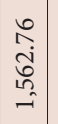 & ○े & 0 & 0 & 0 & $\begin{array}{l}\hat{n} \\
\infty \\
\infty \\
0 \\
0 \\
0\end{array}$ & 0 & 0 & 0 & 0 & 0 & 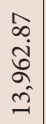 & \\
\hline $\begin{array}{c}\text { əłnoy } \\
\text { əłe!̣doıddeu| }\end{array}$ & 0 & 0 & 0 & 0 & 0 & $\hat{8}$ & กั & 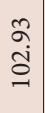 & $\begin{array}{l}\infty \\
\infty \\
\stackrel{0}{0} \\
\infty \\
\end{array}$ & 0 & 0 & 0 & 0 & 0 & 0 & 0 & 0 & 0 & 0 & 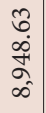 & \\
\hline $\begin{array}{c}\text { əsoด } \\
\text { әૈе!ฺdoıddeu| }\end{array}$ & 0 & 0 & 0 & $\begin{array}{l}\stackrel{n}{n} \\
\stackrel{n}{n}\end{array}$ & 0 & 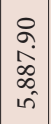 & 0 & 0 & 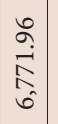 & 0 & 0 & 0 & 0 & 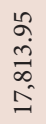 & 0 & 0 & 0 & 0 & 0 & $\begin{array}{l}0 \\
-1 \\
+ \\
0 \\
0 \\
0 \\
0\end{array}$ & \\
\hline $\begin{array}{l}\text { ио!̨ерптеэ } \\
\text { әбеsоa }\end{array}$ & 0 & 0 & 0 & 0 & 0 & 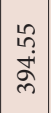 & 0 & 0 & $\begin{array}{c}0 \\
\hat{i} \\
\stackrel{0}{0} \\
\stackrel{2}{\sim}\end{array}$ & 0 & 0 & 0 & 0 & 0 & 0 & 0 & 0 & 0 & 0 & $\begin{array}{l}\vec{n} \\
\hat{n} \\
\tilde{n}\end{array}$ & \\
\hline иo!̣еग!рu!̣яиоо & 0 & 0 & 0 & 0 & 0 & 0 & 0 & 0 & 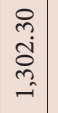 & $\stackrel{\circ}{\circ}$ & 0 & 0 & 0 & 0 & 0 & 0 & 0 & 0 & 0 & 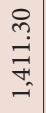 & \\
\hline 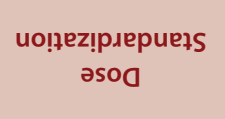 & 0 & 0 & 0 & $\begin{array}{l}n \\
\tilde{n} \\
m\end{array}$ & 0 & 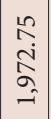 & 0 & 0 & $\begin{array}{c}\vec{J} \\
\stackrel{+}{+} \\
m \\
\hat{i}\end{array}$ & 0 & 0 & 0 & 0 & 0 & 0 & 0 & 0 & 0 & 0 & 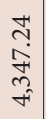 & \\
\hline 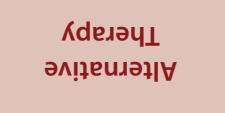 & 0 & 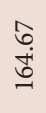 & 0 & $\begin{array}{l}\stackrel{n}{n} \\
\stackrel{n}{m}\end{array}$ & 0 & \begin{tabular}{|l|}
$\tilde{n}$ \\
$\tilde{\rho}$ \\
$\tilde{m}$
\end{tabular} & 눙 & 0 & 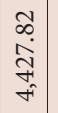 & 。 & 0 & 0 & 0 & 0 & 0 & 0 & 0 & 0 & 0 & 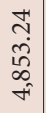 & $\stackrel{\substack{n \\
y}}{a}$ \\
\hline 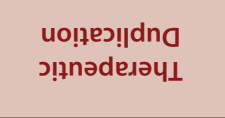 & 0 & 0 & 0 & 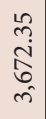 & 0 & 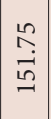 & $\begin{array}{l}\stackrel{n}{n} \\
\stackrel{n}{n}\end{array}$ & 0 & 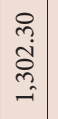 & 0 & 0 & $\begin{array}{l}\infty \\
0 \\
0 \\
+ \\
1 \\
1\end{array}$ & 0 & 0 & 0 & 0 & 0 & 0 & 0 & $\begin{array}{l}m \\
\infty \\
\dot{0} \\
\infty \\
\infty \\
\text { in }\end{array}$ & 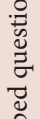 \\
\hline $\begin{array}{c}\text { ио!ฺวеәл } \\
\text { бплр әรләлрн }\end{array}$ & 0 & $\begin{array}{l}\infty \\
\stackrel{2}{a} \\
0 \\
0\end{array}$ & 0 & 0 & 0 & 0 & 0 & 0 & 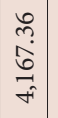 & 0 & 0 & 0 & 0 & 0 & 0 & 0 & 0 & 0 & 0 & 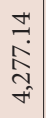 & 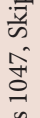 \\
\hline 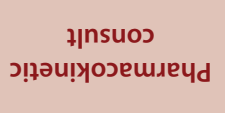 & 0 & 0 & 0 & 0 & 0 & 0 & 0 & 0 & 0 & 0 & 0 & 0 & 0 & 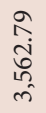 & 0 & 0 & 0 & 0 & 0 & 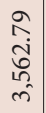 & 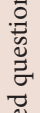 \\
\hline 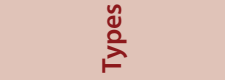 & $\Xi$ & $\cong$ & సూ & $\vec{\sim}$ & $\breve{N}$ & $\vec{\sim}$ & $\tilde{\sim}$ & $\overleftrightarrow{\sim}$ & $\tilde{m}$ & ले & $\breve{m}$ & $\mathscr{F}$ & f & y & 宗 & เீ็ & in & นึ & 0 & 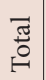 & 童 \\
\hline
\end{tabular}




\begin{tabular}{|c|c|c|c|c|c|c|c|c|c|c|c|c|c|c|c|c|c|c|c|c|c|c|}
\hline \multirow[t]{2}{*}{$\begin{array}{l}\overline{\widetilde{\pi}} \\
\text { চ0 }\end{array}$} & பூ & ?̊. & 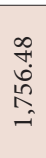 & 8 & 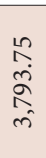 & $\stackrel{8}{\circ}$ & 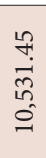 & 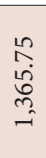 & $\begin{array}{c}\tilde{\sigma} \\
\hat{\sigma} \\
-1\end{array}$ & 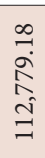 & $\begin{array}{l}8 \\
0 \\
0 \\
0 \\
i\end{array}$ & \&: & 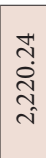 & $\stackrel{8}{0}$ & $\begin{array}{l}2 \\
2 \\
0 \\
10 \\
10 \\
\infty \\
\infty\end{array}$ & $\begin{array}{l}8 \\
0\end{array}$ & $\stackrel{8}{\circ}$ & $\begin{array}{l}8 \\
0\end{array}$ & $\stackrel{8}{0}$ & $\begin{array}{l}8 \\
0 \\
0\end{array}$ & 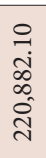 & \\
\hline & 을 & $\infty$ & ๗ै & 0 & $\stackrel{\mathscr{\beth}}{=}$ & 0 & 今े & $\stackrel{2}{+}$ & $m$ & $\stackrel{\mathscr{F}}{\xi}$ & $\stackrel{\sharp}{\sim}$ & 0 & 0 & 0 & $\stackrel{\sim}{\sim}$ & 0 & 0 & 0 & 0 & 0 & 今े & \\
\hline \multirow[t]{2}{*}{$\begin{array}{l}\frac{n}{\varpi} \\
\stackrel{5}{0}\end{array}$} & ப் & 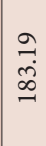 & $\underset{\substack{\sim \\
\infty}}{\stackrel{\infty}{\infty}}$ & $\stackrel{0}{\circ}$ & $\begin{array}{l}\text { m? } \\
\text { @े }\end{array}$ & $\stackrel{0}{\circ}$ & 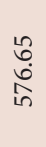 & $\begin{array}{l}\text { 음 } \\
\text { i } \\
\infty \\
-1\end{array}$ & 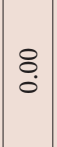 & 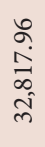 & $\begin{array}{l}8 \\
\dot{8} \\
0 \\
-1\end{array}$ & $\stackrel{0}{\circ}$ & 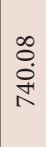 & $\stackrel{8}{0}$ & 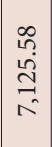 & $\stackrel{0}{0}$ & $\stackrel{8}{0}$ & $\begin{array}{l}0 \\
0\end{array}$ & $\stackrel{0}{0}$ & $\stackrel{8}{0}$ & 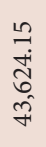 & \\
\hline & 울 & $n$ & $\stackrel{0}{-1}$ & 0 & $\neg$ & 0 & 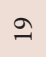 & 0 & 0 & $\stackrel{0}{\sim}$ & $\stackrel{ }{-1}$ & 0 & $N$ & 0 & $d$ & 0 & 0 & 0 & 0 & 0 & $\stackrel{\infty}{-1}$ & \\
\hline \multirow[t]{2}{*}{$\begin{array}{l}\frac{5}{3} \\
0 \\
\frac{5}{5} \\
\frac{5}{5}\end{array}$} & 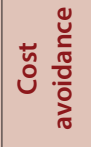 & $\stackrel{8}{0}$ & $\stackrel{8}{\circ}$ & 8 & $\stackrel{8}{\circ}$ & $\stackrel{8}{\circ}$ & $\stackrel{8}{0}$ & $\begin{array}{l}\text { nn } \\
\stackrel{n}{m}\end{array}$ & $\stackrel{8}{0}$ & $\begin{array}{l}\text { کू. } \\
\text { กิ }\end{array}$ & $\stackrel{8}{0}$ & $\stackrel{8}{\circ}$ & $\stackrel{8}{8}$ & $\stackrel{8}{0}$ & $\stackrel{8}{0}$ & $\stackrel{8}{0}$ & $\stackrel{8}{0}$ & $\begin{array}{l}8 \\
0\end{array}$ & 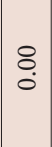 & $\stackrel{8}{0}$ & तิ & \\
\hline & 울 & 0 & 0 & 0 & 0 & 0 & 0 & - & 0 & $\sim$ & 0 & 0 & 0 & 0 & 0 & 0 & 0 & 0 & 0 & 0 & $m$ & \\
\hline \multirow{2}{*}{ 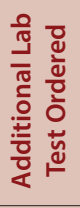 } & 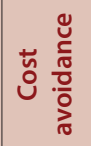 & $\stackrel{8}{0}$ & $\stackrel{8}{\circ}$ & 8 & $\stackrel{8}{\circ}$ & $\stackrel{8}{0}$ & $\stackrel{8}{0}$ & $\stackrel{8}{0}$ & $\begin{array}{l}0 \\
0 \\
0\end{array}$ & $\begin{array}{l}\stackrel{0}{1} \\
\stackrel{+}{+} \\
\hat{0} \\
\infty\end{array}$ & $\begin{array}{l}8 \\
\dot{\hat{\Omega}} \\
1\end{array}$ & $\stackrel{8}{0}$ & 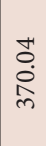 & $\begin{array}{l}8 \\
0 \\
0\end{array}$ & $\begin{array}{l}8 \\
0 \\
0\end{array}$ & $\begin{array}{l}0 \\
0 \\
0\end{array}$ & $\stackrel{8}{0}$ & $\begin{array}{l}8 \\
0\end{array}$ & 8 & $\stackrel{8}{0}$ & 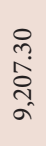 & \\
\hline & 울 & 0 & 0 & 0 & 0 & 0 & 0 & 0 & 0 & $\vec{m}$ & $n$ & 0 & - & 0 & 0 & 0 & 0 & 0 & 0 & 0 & ले & \\
\hline \multirow{2}{*}{ 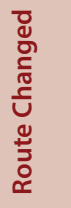 } & 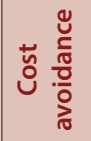 & $\stackrel{0}{0}$ & $\stackrel{8}{\circ}$ & $\stackrel{0}{0}$ & $\stackrel{8}{\circ}$ & $\stackrel{8}{\circ}$ & $\begin{array}{l}R \\
\stackrel{0}{0}\end{array}$ & $\begin{array}{l}10 \\
\stackrel{1}{-1} \\
0 \\
\infty \\
\infty\end{array}$ & $\begin{array}{l}\tilde{\alpha} \\
\hat{\delta} \\
-1\end{array}$ & 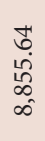 & $\stackrel{0}{0}$ & $\stackrel{8}{\circ}$ & $\stackrel{8}{0}$ & $\stackrel{8}{0}$ & : & $\stackrel{0}{0}$ & $\stackrel{8}{\circ}$ & $\begin{array}{l}8 \\
0\end{array}$ & $\stackrel{8}{0}$ & $\stackrel{8}{0}$ & $\begin{array}{l}\text { अँ } \\
\text { ळे } \\
\alpha \\
\sigma\end{array}$ & \\
\hline & 울 & 0 & 0 & 0 & 0 & 0 & $N$ & নे & $m$ & m & 0 & 0 & 0 & 0 & 0 & 0 & 0 & 0 & 0 & 0 & $\ddot{0}$ & \\
\hline \multirow[t]{2}{*}{ 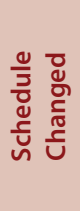 } & گூ & $\stackrel{0}{0}$ & $\begin{array}{l}\infty \\
\text { î } \\
\text { in }\end{array}$ & 8 & \& & $\stackrel{8}{0}$ & 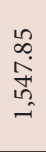 & $\begin{array}{l}\stackrel{n}{n} \\
\text { on } \\
\stackrel{n}{n}\end{array}$ & $\begin{array}{l}8 \\
0 \\
0\end{array}$ & 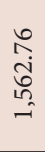 & $\begin{array}{l}8 \\
\dot{0} \\
0\end{array}$ & $\stackrel{8}{0}$ & 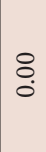 & $\begin{array}{l}8 \\
0 \\
0\end{array}$ & $\mid \begin{array}{l}0 \\
\infty \\
\infty \\
\infty \\
0 \\
0 \\
0 \\
-1\end{array}$ & $\begin{array}{l}8 \\
\vdots \\
0\end{array}$ & $\stackrel{8}{0}$ & $\begin{array}{l}8 \\
0\end{array}$ & 8 & $\begin{array}{l}8 \\
0\end{array}$ & 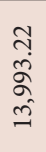 & \\
\hline & 울 & 0 & $\neg$ & 0 & 0 & 0 & $\overrightarrow{i n}$ & $\neg$ & 0 & 0 & - & 0 & 0 & 0 & $m$ & 0 & 0 & 0 & 0 & 0 & $\tilde{6}$ & \\
\hline \multirow{2}{*}{ 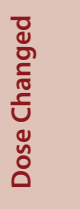 } & பூ & $\begin{array}{l}8 \\
0\end{array}$ & $\begin{array}{l}\infty \\
\text { ஸे } \\
\text { in }\end{array}$ & 8 & $\begin{array}{l}\stackrel{0}{1} \\
8 \\
8\end{array}$ & $\stackrel{8}{0}$ & $\begin{array}{l}\stackrel{n}{\sim} \\
\stackrel{\widetilde{N}}{\Omega} \\
\hat{n}\end{array}$ & $\begin{array}{l}\stackrel{n}{m} \\
\stackrel{n}{m} \\
\tilde{m}\end{array}$ & $\begin{array}{l}0 \\
0 \\
0\end{array}$ & 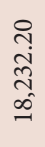 & $\begin{array}{l}8 \\
\dot{0} \\
0\end{array}$ & $\stackrel{8}{0}$ & 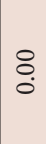 & $\stackrel{8}{0}$ & 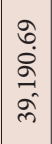 & $\begin{array}{l}8 \\
0 \\
0\end{array}$ & $\begin{array}{l}8 \\
0\end{array}$ & $\begin{array}{l}8 \\
0\end{array}$ & $\begin{array}{l}8 \\
0 \\
0\end{array}$ & 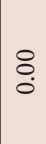 & 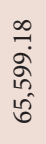 & \\
\hline & 울 & 0 & - & 0 & $N$ & 0 & $\vec{\sim}$ & - & 0 & $\stackrel{R}{R}$ & - & 0 & 0 & 0 & $=$ & 0 & 0 & 0 & 0 & 0 & 今े & \\
\hline \multirow[t]{2}{*}{$\begin{array}{l}\frac{0}{0} \\
\frac{d}{0} \\
\frac{0}{2} \\
\frac{0}{2} \\
\frac{2}{0}\end{array}$} & 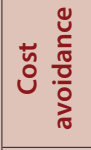 & 8 & $\begin{array}{l}\infty \\
\text { î } \\
\text { in }\end{array}$ & $\stackrel{8}{8}$ & $\stackrel{8}{\circ}$ & $\stackrel{8}{0}$ & 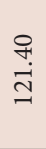 & $\stackrel{8}{\circ}$ & $\begin{array}{l}8 \\
0\end{array}$ & 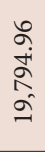 & $\begin{array}{l}8 \\
\text { กิ } \\
\text { ले }\end{array}$ & $\stackrel{8}{\circ}$ & 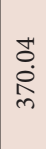 & $\begin{array}{l}8 \\
0 \\
0\end{array}$ & $\begin{array}{l}1 \\
0 \\
\infty \\
\infty \\
0 \\
0 \\
0 \\
0\end{array}$ & $\begin{array}{l}8 \\
0\end{array}$ & $\stackrel{8}{\circ}$ & $\begin{array}{l}8 \\
0\end{array}$ & 8 & 8 & 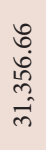 & \\
\hline & 울 & 0 & - & 0 & 0 & 0 & $H$ & 0 & 0 & $\stackrel{0}{\wedge}$ & $m$ & 0 & - & 0 & $m$ & 0 & 0 & 0 & 0 & 0 & $\infty$ & \\
\hline \multirow{2}{*}{ 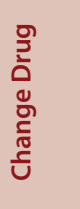 } & பू. & $\begin{array}{l}8 \\
0\end{array}$ & 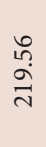 & $\begin{array}{l}8 \\
0 \\
0\end{array}$ & $\stackrel{8}{0}$ & $\stackrel{8}{0}$ & $\begin{array}{l}\stackrel{0}{1} \\
8 \\
0\end{array}$ & $\begin{array}{l}\text { 우 } \\
\underset{\infty}{1} \\
\infty \\
-1\end{array}$ & $\begin{array}{l}0 \\
0 \\
0\end{array}$ & $\begin{array}{l}\stackrel{0}{1} \\
\stackrel{+}{+} \\
\hat{0} \\
\infty\end{array}$ & 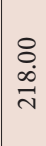 & $\stackrel{8}{\circ}$ & $\begin{array}{l}8 \\
0 \\
0\end{array}$ & $\stackrel{8}{0}$ & $\begin{array}{l}8 \\
0\end{array}$ & $\begin{array}{l}8 \\
0 \\
0\end{array}$ & $\begin{array}{l}8 \\
0\end{array}$ & $\begin{array}{l}8 \\
0\end{array}$ & 8 & 8 & $\begin{array}{l}\widehat{\sigma} \\
\stackrel{+}{\circ} \\
\hat{\infty} \\
\infty\end{array}$ & \\
\hline & 울 & 0 & $r$ & 0 & 0 & 0 & $N$ & 0 & 0 & $\vec{m}$ & $\sim$ & 0 & 0 & 0 & 0 & 0 & 0 & 0 & 0 & 0 & \& & \\
\hline \multirow{2}{*}{ 일 } & 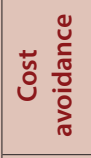 & 궁 & $\begin{array}{l}\overrightarrow{0} \\
\stackrel{+}{+} \\
\text { + }\end{array}$ & $\stackrel{0}{\circ}$ & $\begin{array}{l}\stackrel{2}{\hat{i}} \\
\text { } \\
\stackrel{\text { m}}{2}\end{array}$ & $\stackrel{8}{\circ}$ & $\begin{array}{l}\stackrel{8}{\infty} \\
\stackrel{\text { I }}{\sim}\end{array}$ & 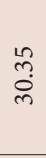 & $\stackrel{0}{0}$ & 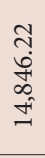 & 8 & $\stackrel{0}{\circ}$ & $\begin{array}{l}\infty \\
0 \\
0 \\
\stackrel{+}{1} \\
1\end{array}$ & $\stackrel{8}{\circ}$ & $\begin{array}{l}n \\
2 \\
2 \\
0 \\
\infty \\
\\
\end{array}$ & $\begin{array}{l}8 \\
0 \\
0\end{array}$ & $\stackrel{8}{\circ}$ & $\begin{array}{l}0 \\
0 \\
0\end{array}$ & 8 & $\begin{array}{l}8 \\
0 \\
0\end{array}$ & \begin{tabular}{l}
$\infty$ \\
\} $\\
{\infty} \\
{\infty} \\
{\hat{n}}$ & $\frac{n}{\stackrel{n}{\circ}}$ \\
\hline & 울 & - & $a$ & 0 & తี & 0 & $\infty$ & - & 0 & in & 0 & 0 & $N$ & 0 & in & 0 & 0 & 0 & 0 & 0 & $\stackrel{\text { ڤn }}{\mathrm{N}}$ & e \\
\hline$\stackrel{\circlearrowright}{凶}$ & 2 & $\stackrel{\pi}{-1}$ & $\Rightarrow$ & กี & $\hat{\text { กิ }}$ & $\ddot{\sim}$ & $\vec{\sim}$ & $\stackrel{\sim}{\sim}$ & $\overleftrightarrow{\sim}$ & mూ & ले & $\stackrel{n}{n}$ & F & f & $y$ & Z्F & กี & in & in & 6 & సّٓँّ & 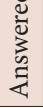 \\
\hline
\end{tabular}
\end{tabular}




\begin{tabular}{|c|c|c|c|c|c|c|c|c|c|c|c|c|c|c|c|c|c|c|c|c|c|c|}
\hline \multirow[t]{2}{*}{ 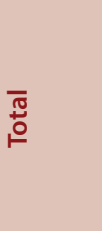 } & பั & ల̊ & $\begin{array}{l}\infty \\
\stackrel{\infty}{+} \\
6 \\
\stackrel{1}{10} \\
\end{array}$ & $\stackrel{8}{\circ}$ & 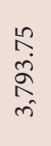 & $\stackrel{8}{0}$ & 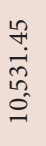 & $\begin{array}{l}n \\
2 \\
10 \\
0 \\
m \\
-1\end{array}$ & $\begin{array}{l}\tilde{\alpha} \\
\tilde{\delta} \\
\hat{-}\end{array}$ & $\begin{array}{l}\infty \\
\stackrel{-}{2} \\
\hat{\leqslant} \\
\hat{\Xi} \\
\Xi\end{array}$ & $\begin{array}{l}8 \\
\dot{0} \\
6 \\
i \\
i\end{array}$ & $\begin{array}{l}0 \\
0 \\
0\end{array}$ & 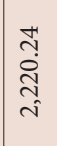 & $\stackrel{8}{0}$ & $\begin{array}{l}0 \\
0 \\
6 \\
0 \\
10 \\
10 \\
\infty\end{array}$ & $\stackrel{8}{0}$ & $\stackrel{8}{0}$ & $\stackrel{8}{\circ}$ & $\stackrel{8}{0}$ & $\stackrel{8}{0}$ & 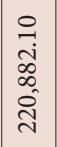 & \\
\hline & 울 & $\infty$ & $\tilde{m}$ & 0 & $\stackrel{\mathscr{2}}{=}$ & 0 & 今े & 孚 & $m$ & $\tilde{m}$ & $\stackrel{\Delta}{\sim}$ & 0 & 0 & 0 & $\stackrel{\Delta}{\sim}$ & 0 & 0 & 0 & 0 & 0 & f & \\
\hline \multirow{2}{*}{$\begin{array}{l}\frac{5}{3} \\
0 \\
\frac{\mathfrak{z}}{c} \\
\text { c }\end{array}$} & 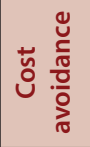 & जे & $\begin{array}{l}2 \\
\text { के } \\
\text { ம் }\end{array}$ & $\stackrel{0}{\circ}$ & $\begin{array}{l}\tilde{\omega} \\
\ddot{b} \\
\stackrel{0}{0} \\
\dot{m}\end{array}$ & $\stackrel{8}{0}$ & $\begin{array}{l}2 \\
\alpha \\
\infty \\
\hat{\alpha} \\
\hat{\omega}\end{array}$ & 곳 & $\begin{array}{l}\tilde{O} \\
0 \\
0 \\
0\end{array}$ & $\begin{array}{c}\tilde{N} \\
\infty \\
\infty \\
+ \\
0 \\
-0 \\
-1\end{array}$ & $\begin{array}{l}8 \\
\dot{2} \\
8 \\
0 \\
-1\end{array}$ & $\stackrel{8}{0}$ & 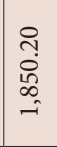 & $\stackrel{0}{0}$ & $\begin{array}{l}2 n \\
2 \\
n \\
\infty \\
\\
\end{array}$ & $\stackrel{0}{0}$ & $\stackrel{8}{0}$ & $\stackrel{0}{0}$ & $\stackrel{0}{0}$ & $\stackrel{0}{0}$ & 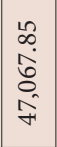 & \\
\hline & 울 & $\neg$ & $\neg$ & 0 & $\overrightarrow{0}$ & 0 & $\hat{\sigma}$ & $\tilde{\omega}$ & $N$ & $\widetilde{\sigma}$ & $\stackrel{ }{-}$ & 0 & in & 0 & in & 0 & 0 & 0 & 0 & 0 & $\underset{7}{7}$ & \\
\hline \multirow{2}{*}{ 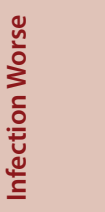 } & 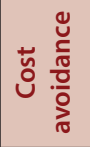 & $\stackrel{8}{0}$ & $\stackrel{0}{0}$ & $\stackrel{0}{\circ}$ & ¿ & $\stackrel{8}{0}$ & $\stackrel{8}{\circ}$ & $\stackrel{0}{0}$ & $\begin{array}{l}0 \\
0\end{array}$ & 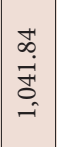 & $\begin{array}{l}8 \\
\dot{g} \\
0\end{array}$ & $\stackrel{0}{0}$ & $\stackrel{0}{0}$ & $\begin{array}{l}0 \\
0 \\
0\end{array}$ & $\stackrel{8}{0}$ & $\stackrel{0}{0}$ & $\stackrel{8}{0}$ & ¿ & $\stackrel{0}{0}$ & $\stackrel{0}{0}$ & $\begin{array}{l}+1 \\
0 \\
0 \\
10 \\
-1 \\
-1\end{array}$ & \\
\hline & 울 & 0 & 0 & 0 & 0 & 0 & 0 & 0 & 0 & 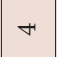 & - & 0 & 0 & 0 & 0 & 0 & 0 & 0 & 0 & 0 & in & \\
\hline \multirow{2}{*}{ 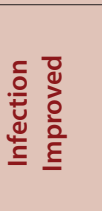 } & 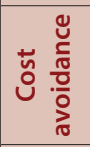 & 8 & $\begin{array}{l}\text { ले } \\
\text { సें }\end{array}$ & $\stackrel{8}{0}$ & $\begin{array}{l}\stackrel{0}{1} \\
\dot{\infty} \\
\infty \\
-\end{array}$ & $\stackrel{8}{0}$ & 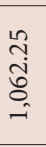 & $\begin{array}{l}n \\
\tilde{n} \\
0 \\
\tilde{m}\end{array}$ & $\begin{array}{l}8 \\
0\end{array}$ & $\begin{array}{l}0 \\
0 \\
0 \\
0 \\
+1 \\
\text { in }\end{array}$ & $\begin{array}{l}8 \\
\infty \\
\infty \\
\vec{N}\end{array} \mid$ & $\stackrel{8}{0}$ & $\stackrel{8}{0}$ & $\begin{array}{l}8 \\
0\end{array}$ & $\begin{array}{l}\hat{\imath} \\
\hat{i} \\
1 \\
m \\
m\end{array}$ & $\stackrel{8}{0}$ & $\stackrel{8}{0}$ & 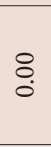 & $\stackrel{8}{0}$ & $\stackrel{8}{0}$ & 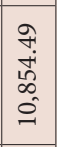 & \\
\hline & 울 & 0 & 6 & 0 & 0 & 0 & $\stackrel{m}{m}$ & - & 0 & $\vec{\sim}$ & $\mathrm{N}$ & 0 & 0 & 0 & $\neg$ & 0 & 0 & 0 & 0 & 0 & $N$ & \\
\hline \multirow{2}{*}{ 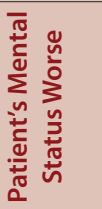 } & 芯 $\frac{\stackrel{0}{\frac{c}{\pi}}}{\frac{0}{0}}$ & 8 & $\stackrel{8}{0}$ & $\stackrel{8}{0}$ & \& & $\stackrel{8}{\circ}$ & $\begin{array}{l}m \\
\tilde{m} \\
\tilde{m}^{n}\end{array}$ & $\begin{array}{l}8 \\
0\end{array}$ & $\begin{array}{l}8 \\
0 \\
0\end{array}$ & $\begin{array}{l}8 \\
0 \\
0\end{array}$ & 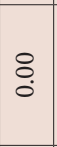 & 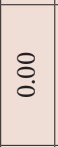 & $\stackrel{8}{0}$ & 8 & $\stackrel{8}{0}$ & $\begin{array}{l}8 \\
0\end{array}$ & $\stackrel{8}{0}$ & $\stackrel{8}{8}$ & $\stackrel{8}{\circ}$ & $\stackrel{0}{0}$ & $\begin{array}{l}n \\
m \\
0 \\
\dot{m}\end{array}$ & \\
\hline & 울 & 0 & 0 & 0 & 0 & 0 & $\neg$ & 0 & 0 & 0 & 0 & 0 & 0 & 0 & 0 & 0 & 0 & 0 & 0 & 0 & - & \\
\hline \multirow{2}{*}{ 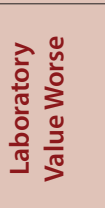 } & 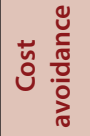 & 8 & $\begin{array}{l}8 \\
0\end{array}$ & $\stackrel{8}{0}$ & \& & $\stackrel{8}{0}$ & $\stackrel{8}{\circ}$ & $\begin{array}{l}8 \\
0\end{array}$ & $\begin{array}{l}8 \\
0\end{array}$ & 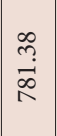 & $\begin{array}{l}\stackrel{8}{0} \\
\dot{g} \\
0\end{array}$ & $\stackrel{8}{8}$ & $\stackrel{8}{0}$ & $\stackrel{8}{0}$ & $\stackrel{8}{0}$ & $\stackrel{8}{0}$ & $\stackrel{8}{0}$ & \& & $\stackrel{8}{0}$ & $\stackrel{8}{0}$ & $\begin{array}{l}\infty \\
m \\
\stackrel{2}{2} \\
\infty\end{array}$ & \\
\hline & o & 0 & 0 & 0 & 0 & 0 & 0 & 0 & 0 & $m$ & $\neg$ & 0 & 0 & 0 & 0 & 0 & 0 & 0 & 0 & 0 & $H$ & \\
\hline \multirow{2}{*}{ 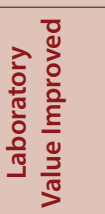 } & பั & जे & 8 & $\stackrel{8}{0}$ & $\stackrel{8}{0}$ & 8 & $\begin{array}{l}\stackrel{8}{ } \\
\stackrel{\text { I }}{\text { J }}\end{array}$ & $\begin{array}{l}8 \\
0\end{array}$ & $\begin{array}{l}8 \\
0 \\
0\end{array}$ & 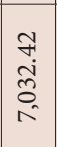 & $\begin{array}{l}8 \\
\dot{a} \\
0 \\
0\end{array}$ & 8 & $\stackrel{8}{0}$ & 8 & 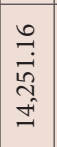 & $\begin{array}{l}8 \\
0 \\
0\end{array}$ & $\stackrel{8}{0}$ & $\stackrel{8}{0}$ & $\stackrel{8}{0}$ & 8 & 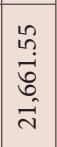 & \\
\hline & 울 & - & 0 & 0 & 0 & 0 & $\infty$ & 0 & 0 & $\widehat{\sim}$ & $\neg$ & 0 & 0 & 0 & $H$ & 0 & 0 & 0 & 0 & 0 & F & \\
\hline \multirow[t]{2}{*}{ 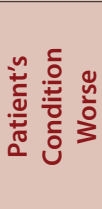 } & U气 & $\stackrel{8}{0}$ & 8 & $\stackrel{8}{\circ}$ & $\stackrel{8}{\circ}$ & $\stackrel{0}{0}$ & $\stackrel{8}{0}$ & $\stackrel{8}{0}$ & 8 & 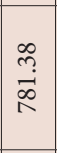 & $\stackrel{8}{0}$ & 8 & $\stackrel{8}{\circ}$ & $\stackrel{8}{0}$ & $\stackrel{8}{0}$ & $\stackrel{8}{0}$ & $\stackrel{8}{\circ}$ & $\stackrel{8}{\circ}$ & $\stackrel{8}{\circ}$ & 8 & 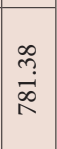 & \\
\hline & 울 & 0 & 0 & 0 & 0 & 0 & 0 & 0 & 0 & $m$ & 0 & 0 & 0 & 0 & 0 & 0 & 0 & 0 & 0 & 0 & $m$ & \\
\hline \multirow{2}{*}{ 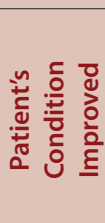 } & 芯 $\frac{\stackrel{\Xi}{\frac{c}{\pi}}}{\frac{0}{0}}$ & $\stackrel{8}{8}$ & $\begin{array}{l}\overrightarrow{5} \\
\dot{+} \\
\dot{f}\end{array}$ & $\stackrel{8}{8}$ & \begin{tabular}{l}
$\stackrel{2}{1}$ \\
\multirow{2}{n}{} \\
$\stackrel{2}{9}$
\end{tabular} & $\begin{array}{l}8 \\
0\end{array}$ & 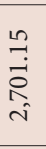 & $\begin{array}{c}0 \\
-1 \\
\infty \\
\infty \\
-1\end{array}$ & $\vec{m}$ & 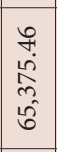 & $\begin{array}{c}8 \\
\text { in } \\
\text { in }\end{array}$ & 8 & $\begin{array}{l}\vec{H} \\
\dot{0} \\
\stackrel{m}{m}\end{array}$ & 8 & 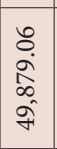 & 8 & $\stackrel{8}{0}$ & $\stackrel{8}{0}$ & $\stackrel{8}{0}$ & 8 & 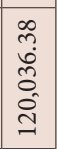 & \\
\hline & 울 & 0 & $a$ & 0 & $\stackrel{2}{2}$ & 0 & ळे & 0 & - & $\overrightarrow{\stackrel{n}{n}}$ & in & 0 & $\rightarrow$ & 0 & $\exists$ & 0 & 0 & 0 & 0 & 0 & बे & \\
\hline \multirow{2}{*}{ 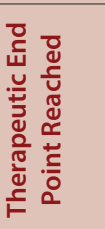 } & பั & $\begin{array}{l}\text { ô. } \\
\text { in } \\
\text { in }\end{array}$ & 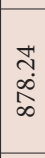 & $\stackrel{8}{\circ}$ & $\begin{array}{l}\text { 우 } \\
\text { बें }\end{array}$ & $\stackrel{8}{\circ}$ & $\begin{array}{l}20 \\
\alpha \\
i n \\
i n\end{array}$ & $\begin{array}{c}0 \\
\underset{1}{1} \\
\infty \\
-1\end{array}$ & $\begin{array}{l}8 \\
0 \\
0\end{array}$ & 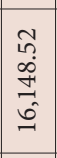 & $\begin{array}{l}8 \\
0 \\
\dot{0} \\
\ddot{H}\end{array}$ & 8 & $\stackrel{8}{0}$ & 8 & 8 & $\stackrel{8}{0}$ & $\stackrel{8}{0}$ & 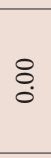 & $\stackrel{8}{0}$ & 8 & $\begin{array}{l}\infty \\
\infty \\
\infty \\
\infty \\
0+1 \\
\infty \\
\infty \\
-1\end{array}$ & 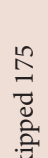 \\
\hline & 운 & 6 & $\because$ & 0 & $m$ & 0 & $\simeq$ & 6 & 0 & $\widetilde{\sigma}$ & $H$ & 0 & 0 & 0 & 0 & 0 & 0 & 0 & 0 & 0 & $\Xi$ & 犬े \\
\hline$\stackrel{\times}{\stackrel{x}{\sim}}$ & ํㅜㄴ & $\pi$ & $=$ & तี & ते & $\stackrel{\sim}{\sim}$ & $\vec{N}$ & 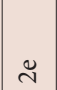 & $\breve{\sim}$ & $\tilde{m}$ & m & $\breve{m}$ & F & f & y & F & ก็ & in & $\breve{n}$ & 0 & 㺃 & 岂 \\
\hline
\end{tabular}




\begin{tabular}{|c|c|c|c|c|c|c|c|c|c|c|c|c|c|c|c|c|c|c|c|c|c|c|c|c|}
\hline \multirow{3}{*}{$\circ$} & 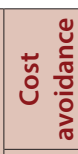 & $\begin{array}{l}\text { mె } \\
\text { בे }\end{array}$ & 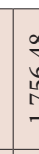 & 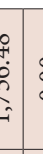 & $\stackrel{8}{8}$ & 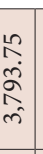 & $\begin{array}{l}8 \\
0 \\
0\end{array}$ & 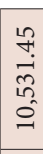 & 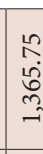 & $\begin{array}{l}\tilde{o} \\
\hat{\delta}\end{array}$ & $\begin{array}{l}\infty \\
\frac{1}{2} \\
1 \\
\end{array}$ & $?$ & & ¿ֶ. & 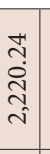 & $\stackrel{8}{0}$ & $\begin{array}{l}2 \\
0 \\
0 \\
0 \\
10 \\
\infty \\
\infty\end{array}$ & $\stackrel{8}{0}$ & $\begin{array}{l}8 \\
\circ \\
0\end{array}$ & $\begin{array}{l}8 \\
\circ\end{array}$ & $\begin{array}{l}8 \\
\circ\end{array}$ & $\stackrel{8}{\circ}$ & 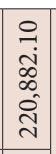 & \\
\hline & $\therefore$ & छे & छેे & b. & ס̊ & ذి & $\begin{array}{l}\text { ठें } \\
\text { ठे }\end{array}$ & 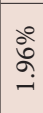 & ठें & ఏे & 4 & & & 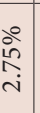 & ذి & 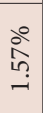 & 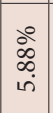 & $\mid \begin{array}{l}\circ \\
6 \\
0 \\
0\end{array}$ & $\begin{array}{c}\text { iे } \\
\text { †े } \\
i\end{array}$ & $\begin{array}{l}\text { ठें } \\
\stackrel{1}{\wedge}\end{array}$ & $\begin{array}{l}\stackrel{\circ}{\circ} \\
\stackrel{-}{\circ}\end{array}$ & $\begin{array}{l}\text { ठें } \\
\dot{0}\end{array}$ & 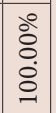 & \\
\hline & $\stackrel{0}{z}$ & $\infty$ & व & s. & o & 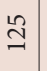 & 0 & मे & \& & m & $\tilde{y}$ & & & 0 & 0 & 0 & $\underset{\sim}{\sim}$ & 0 & 0 & 0 & 0 & 0 & ثิ으 & \\
\hline \multirow{3}{*}{ 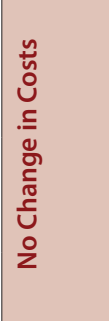 } & نे & $\stackrel{8}{\vdots}$ & 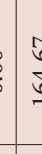 & & $\begin{array}{l}8 \\
\\
\end{array}$ & 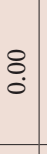 & $\underset{+}{8}$ & 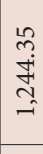 & $\begin{array}{c}\stackrel{0}{1} \\
\stackrel{\infty}{\sim}\end{array}$ & $\bar{m}$ & శ్ & 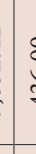 & & \begin{tabular}{l}
8 \\
\hdashline \\
$\circ$
\end{tabular} & $\begin{array}{l}8 \\
:\end{array}$ & $\stackrel{8}{0}$ & \begin{tabular}{l}
8 \\
\hdashline
\end{tabular} & $\stackrel{8}{0}$ & \begin{tabular}{l}
8 \\
\hdashline
\end{tabular} & \begin{tabular}{l}
8 \\
\hdashline
\end{tabular} & $\begin{array}{l}8 \\
\circ\end{array}$ & $\stackrel{8}{0}$ & $\begin{array}{l}1 \\
\infty \\
\alpha \\
\hat{D} \\
\sigma \\
\sigma\end{array}$ & \\
\hline & $\circ^{\circ}$ & ఫे & વે & 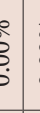 & 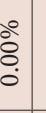 & ذ̊̊̊口̊ & $\begin{array}{l}\text { ठें } \\
0 \\
0\end{array}$ & ذٌ & ठें & वे & 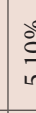 & & & 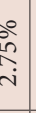 & $\begin{array}{l}\text { ذి } \\
\text { वें } \\
\text { in }\end{array}$ & 齐 & $\begin{array}{l}\infty \\
\infty \\
\infty \\
\text { ஸे }\end{array}$ & $\begin{array}{l}\text { बें } \\
\hat{b} \\
0\end{array}$ & 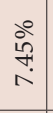 & 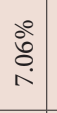 & $\begin{array}{l}\text { 今े } \\
\stackrel{\circ}{-}\end{array}$ & 客 & $\begin{array}{l}\stackrel{0}{0} \\
\dot{0} \\
\dot{0}\end{array}$ & \\
\hline & $\stackrel{0}{z}$ & $\circ$ & $\sim$ & n & 。 & 0 & 0 & $\exists$ & 6 & - & t & & & 0 & 0 & 0 & 0 & 0 & 0 & 0 & 0 & 0 & $\infty$ & \\
\hline \multirow{3}{*}{ 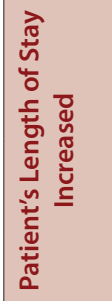 } & 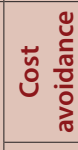 & ¿ & 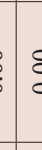 & : & 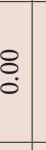 & $\begin{array}{l}8 \\
\vdots \\
\vdots\end{array}$ & $\begin{array}{l}8 \\
0\end{array}$ & $\stackrel{8}{0}$ & $\stackrel{8}{8}$ & $\stackrel{8}{8}$ & $g$ & & & \begin{tabular}{l}
8 \\
\hdashline \\
\end{tabular} & $\begin{array}{l}8 \\
\vdots \\
\end{array}$ & $\stackrel{8}{0}$ & \begin{tabular}{l}
8 \\
\hdashline
\end{tabular} & $\begin{array}{l}8 \\
0 \\
\end{array}$ & $\stackrel{8}{\circ}$ & $\begin{array}{l}8 \\
\circ\end{array}$ & $\begin{array}{l}8 \\
0 \\
\end{array}$ & $\stackrel{8}{0}$ & $\begin{array}{c}0 \\
0 \\
0 \\
\vec{c}\end{array}$ & \\
\hline & $2^{\circ}$ & ఏे & aे & : & 葛 & ذ̊ें & $\begin{array}{l}\text { ذें } \\
\vdots \\
\end{array}$ & ठें & ذें & $\begin{array}{l}\text { ఏे } \\
\text { वे }\end{array}$ & ฉి & & & 范 & 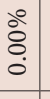 & $\begin{array}{l}\text { ذें } \\
\vdots \\
0\end{array}$ & $\begin{array}{l}\text { oे } \\
\vdots \\
0\end{array}$ & $\begin{array}{l}\text { oें } \\
\text { ठे } \\
\end{array}$ & $\begin{array}{l}\text { ذें } \\
\vdots \\
0\end{array}$ & $\begin{array}{l}\text { 今े } \\
\vdots \\
0\end{array}$ & $\begin{array}{l}0 \\
\vdots \\
0 \\
0\end{array}$ & $\begin{array}{l}\text { oे } \\
\text { oे } \\
\end{array}$ & $\begin{array}{c}0 \\
2 \\
0\end{array}$ & \\
\hline & 울 & $\circ$ & 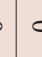 & o & 。 & 0 & 0 & 0 & ○ & 0 & 0 & & & 0 & 0 & 0 & 0 & 0 & 0 & 0 & 0 & 0 & $\mathrm{~N}$ & \\
\hline \multirow{3}{*}{ 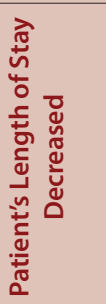 } & 苞 & $\stackrel{8}{\circ}$ & $\varepsilon$ & : & 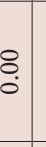 & \begin{tabular}{l}
8 \\
$\vdots$ \\
\hdashline
\end{tabular} & $\begin{array}{l}8 \\
:\end{array}$ & $\stackrel{8}{\circ}$ & $\stackrel{8}{\circ}$ & $\stackrel{8}{\circ}$ & $\bar{a}$ & & & $\begin{array}{l}8 \\
\vdots \\
0\end{array}$ & $\begin{array}{l}8 \\
0 \\
0\end{array}$ & $\stackrel{8}{0}$ & $\begin{array}{l}\overrightarrow{7} \\
\hat{b} \\
0 \\
\hat{m} \\
0\end{array}$ & $\begin{array}{l}8 \\
0 \\
0\end{array}$ & $\begin{array}{l}8 \\
0 \\
0\end{array}$ & $\begin{array}{l}0 \\
0 \\
0\end{array}$ & $\begin{array}{l}8 \\
0 \\
0\end{array}$ & $\stackrel{0}{0}$ & $\begin{array}{l}\hat{T} \\
0 \\
0 \\
0 \\
0 \\
\tilde{j} \\
\end{array}$ & \\
\hline & $\circ^{\circ}$ & ఏें & aे & 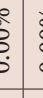 & : & ذి̊ & $\begin{array}{l}\text { ڤें } \\
\dot{0}\end{array}$ & 今े & ذें & ఏे & 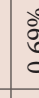 & & & : & 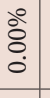 & $\begin{array}{l}\text { ठें } \\
\text { ठे. }\end{array}$ & 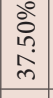 & 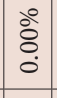 & $\begin{array}{l}\text { 今े } \\
\dot{0} \\
0\end{array}$ & $\begin{array}{l}\text { 今ें } \\
0 \\
0\end{array}$ & $\begin{array}{l}0 \\
\text { oे } \\
\dot{0}\end{array}$ & oें & i̊ & \\
\hline & $\stackrel{0}{2}$ & ○ & . & o & 。 & 0 & 0 & 0 & ○ & 0 & r & & & 0 & ○ & 0 & $a$ & 0 & 0 & 0 & 0 & 0 & $\simeq$ & \\
\hline \multirow{3}{*}{ 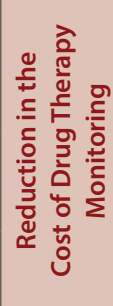 } & نั & ¿ & 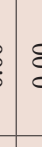 & 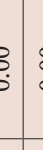 & $\begin{array}{l}8 \\
0 \\
0\end{array}$ & $\begin{array}{l}\stackrel{R}{0} \\
\dot{8}\end{array}$ & $\stackrel{8}{\circ}$ & $\begin{array}{l}\text { है } \\
\dot{a}\end{array}$ & $\begin{array}{l}\stackrel{0}{0} \\
\text { s. }\end{array}$ & ¿ & 8 & 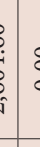 & & $\begin{array}{l}\stackrel{8}{0} \\
\vdots\end{array}$ & $\begin{array}{l}8 \\
\vdots\end{array}$ & $\begin{array}{l}8 \\
0\end{array}$ & $\stackrel{8}{0}$ & $\begin{array}{l}8 \\
0 \\
0\end{array}$ & $\stackrel{8}{\circ}$ & $\begin{array}{l}0 \\
0 \\
0\end{array}$ & $\stackrel{8}{\circ}$ & $\stackrel{8}{0}$ & $\begin{array}{c}n \\
0 \\
0 \\
0 \\
0 \\
i \\
i\end{array}$ & \\
\hline & $\therefore$ & ذे & ฉે & ن. & 苍 & 客 & 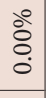 & $\begin{array}{l}\text { ळे } \\
\infty \\
0 \\
0\end{array}$ & I্ं & ఏे & $\frac{\partial}{\mathrm{c}}$ & & 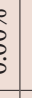 & هั̊ & 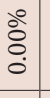 & $\begin{array}{l}\text { ठें } \\
\text { ठै. }\end{array}$ & $\begin{array}{l}\text { oे } \\
\text { ठे }\end{array}$ & 㝘 & ठ̊̊. & $\begin{array}{l}\text { oे } \\
\vdots \\
0\end{array}$ & ठ̊̀ & $\begin{array}{l}0 \\
\text { ठे } \\
0\end{array}$ & 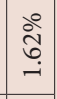 & \\
\hline & z & 。 & 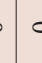 & o & 。 & $\sim$ & 0 & $m$ & $\mathrm{~N}$ & 0 & 9 & & & 0 & 0 & 0 & 0 & 0 & 0 & 0 & 0 & 0 & $\approx$ & \\
\hline \multirow{3}{*}{ 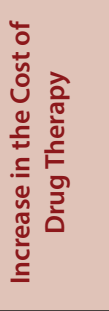 } & 苞 & $\begin{array}{l}7 \\
0\end{array}$ & 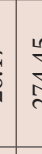 & $\begin{array}{l}f \\
\text { fi } \\
\text { ti }\end{array}$ & $\begin{array}{l}8 \\
\\
\end{array}$ & $\begin{array}{l}\hat{R} \\
\dot{S} \\
\dot{b}\end{array}$ & $\stackrel{8}{\circ}$ & 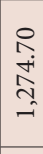 & ભొ & व & a & & & \begin{tabular}{l}
8 \\
\hdashline \\
\hdashline
\end{tabular} & 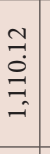 & $\stackrel{8}{\circ}$ & $\begin{array}{l}8 \\
0 \\
\end{array}$ & $\begin{array}{l}8 \\
0 \\
0\end{array}$ & $\begin{array}{l}8 \\
0\end{array}$ & $\begin{array}{l}8 \\
0 \\
0\end{array}$ & $\begin{array}{l}8 \\
0 \\
0\end{array}$ & $\begin{array}{l}8 \\
0 \\
\end{array}$ & $\begin{array}{l}n \\
0 \\
\infty \\
0 \\
\mathfrak{g} \\
\mathfrak{g}\end{array}$ & \\
\hline & $\therefore$ & 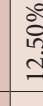 & ปั & . & ठ̊े & 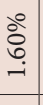 & $\begin{array}{l}\text { ठें } \\
\text { ठे }\end{array}$ & 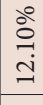 & ذి సે & ठे & बें & & & $\begin{array}{l}\text { ồ } \\
\text { ¿ } \\
0\end{array}$ & $\begin{array}{l}\text { oे } \\
0 \\
\dot{0} \\
\text { in }\end{array}$ & $\begin{array}{l}\text { oे } \\
\text { ठे. } \\
\end{array}$ & $\begin{array}{l}0 \\
\vdots \\
0 \\
0\end{array}$ & 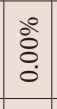 & $\begin{array}{l}\text { ठें } \\
\text { ठ }\end{array}$ & $\begin{array}{l}\text { ठें } \\
\dot{0}\end{array}$ & oे̀ & $\begin{array}{l} \\
\text { ठें } \\
0\end{array}$ & $\begin{array}{l}\text { ذें } \\
\text { ă } \\
\end{array}$ & 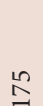 \\
\hline & 울 & - & 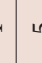 & م & 。 & $\mathrm{N}$ & 0 & ₹ & - & 0 & $\bar{\pi}$ & & & 0 & $m$ & 0 & 0 & 0 & 0 & 0 & 0 & 0 & a & 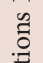 \\
\hline \multirow{3}{*}{ 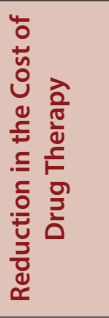 } & 仓ั & $\stackrel{\infty}{\infty}$ & 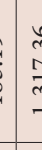 & ?: & $\begin{array}{l}8 \\
0 \\
\end{array}$ & 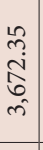 & $\stackrel{0}{0}$ & 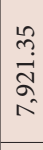 & $\begin{array}{l}\text { ठ } \\
\text { d. } \\
\text { d. } \\
-\end{array}$ & $\begin{array}{l}0 \\
0 \\
0 \\
0\end{array}$ & 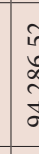 & 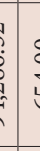 & & \begin{tabular}{l}
8 \\
\hdashline \\
0
\end{tabular} & $\begin{array}{l}\mathcal{T} \\
\stackrel{0}{0} \\
= \\
\approx \\
\approx\end{array}$ & $\begin{array}{l}8 \\
\circ \\
\end{array}$ & 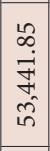 & 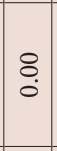 & $\stackrel{8}{0}$ & $\stackrel{8}{0}$ & $\begin{array}{l}0 \\
0 \\
0\end{array}$ & $\stackrel{8}{0}$ & $\begin{array}{l}2 \\
2 \\
\hat{1} \\
\hat{0} \\
0 \\
0\end{array}$ & 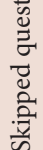 \\
\hline & ○ & in & $i$ & مُ & هُ & 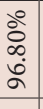 & 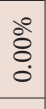 & 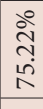 & $\begin{array}{l}\text { ذें } \\
\text { ¿ें }\end{array}$ & $\begin{array}{l}\text { Dे } \\
\text { bे } \\
\text { d }\end{array}$ & $\propto$ & & & ל̊. & $\begin{array}{l}\text { ¿ें } \\
\text { ¿ें } \\
\text { in }\end{array}$ & $\begin{array}{l}\text { ठें } \\
\text { ठे. }\end{array}$ & 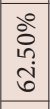 & $\begin{array}{l}\text { oे̀ } \\
0 \\
0\end{array}$ & 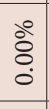 & $\begin{array}{l}\text { oें } \\
0 \\
0\end{array}$ & $\begin{array}{l}\text { Oें } \\
\dot{0} \\
\end{array}$ & oें & $\begin{array}{l}\text { के } \\
\text { ๙े } \\
\text { }\end{array}$ & के \\
\hline & $\stackrel{\circ}{z}$ & 工 & 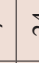 & ث & 。 & $\vec{\Xi}$ & 0 & $\overrightarrow{\mathrm{d}}$ & m & $\tau$ & d़ & 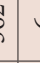 & & 0 & $m$ & 0 & $\stackrel{2}{2}$ & 0 & 0 & 0 & 0 & 0 & $\hat{\infty}$ & 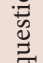 \\
\hline$\stackrel{\times}{ٍ}$ & $\stackrel{0}{z}$ & - & 1 & 2 & तี & $\overrightarrow{\mathrm{\lambda}}$ & 4 & $\pi$ & $\stackrel{\sim}{\sim}$ & 10 & & & & 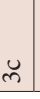 & अ & f & $y$ & 亲 & ก็ & ही & in & 6 & 䒿 & \\
\hline
\end{tabular}


Table 12: Type of cost aviodance used for phramcaist interventions.

\begin{tabular}{|l|c|c|c|}
\hline Answer Options & $\begin{array}{c}\text { Response } \\
\text { Percent }\end{array}$ & $\begin{array}{c}\text { Response } \\
\text { Count }\end{array}$ & Total cost \\
\hline $1 \mathrm{a}$ & $0.76 \%$ & 8 & 209.36 \\
\hline $\mathrm{1b}$ & $3.06 \%$ & 32 & $1,756.48$ \\
\hline $2 \mathrm{a}$ & $0.00 \%$ & 0 & 0.00 \\
\hline 2b & $11.94 \%$ & 125 & $3,793.75$ \\
\hline 2c & $0.00 \%$ & 0 & 0.00 \\
\hline $2 \mathrm{~d}$ & $33.14 \%$ & 347 & $10,531.45$ \\
\hline $2 \mathrm{e}$ & $4.30 \%$ & 45 & $1,365.75$ \\
\hline $2 \mathrm{f}$ & $0.29 \%$ & 3 & 102.93 \\
\hline $3 \mathrm{a}$ & $41.36 \%$ & 433 & $112,779.18$ \\
\hline 3b & $2.29 \%$ & 24 & $2,616.00$ \\
\hline $3 \mathrm{c}$ & $0.00 \%$ & 0 & 0.00 \\
\hline $4 \mathrm{a}$ & $0.57 \%$ & 6 & $2,220.24$ \\
\hline $4 \mathrm{~b}$ & $0.00 \%$ & 0 & 0.00 \\
\hline $4 \mathrm{c}$ & $2.29 \%$ & 24 & $85,506.96$ \\
\hline $4 \mathrm{~d}$ & $0.00 \%$ & 0 & 0.00 \\
\hline $5 \mathrm{a}$ & $0.00 \%$ & 0 & 0.00 \\
\hline $5 \mathrm{~b}$ & $0.00 \%$ & 0 & 0.00 \\
\hline $5 \mathrm{c}$ & $0.00 \%$ & 0 & 0.00 \\
\hline 6 & $0.00 \%$ & 0 & 0.00 \\
\hline answered question & & 1047 & $220,882.10$ \\
\hline skipped question & & 175 & \\
\hline
\end{tabular}

That is similar what reported by Aljbouri TM. ${ }^{12}$ Those medications highly interpreted by the pharmacist at critical care services with highly consequences problems and additional cost if they happened. The highest cost avoidance of type of interventions after other types was inappropriate dosing and TPN consultation. That is related to use of high-risk medications and expecting serious complications with the further incremental hospital of stay and high economic burden. The majority of cost avoidance leas to patients improved conditions that's a clear economic outcome of a pharmacist working at critical care services. The results showed that is most of the cost avoidance due to drug therapy cost related mistake prevention and reduced complications with the sequencing of reduction of hospitals stay. The type of cost calculation used was dosing adjustments related cost because of interventions related change dose or therapy duplication. The critical care pharmacist had a high economic impact of from very simple of pharmacy work-related interventions. We excepted higher cost if we include other services related setting guidelines, answering drug information inquiries and patients counseling services. The proving of a clinical pharmacist to critical care services at a private hospital is highly recommended in Riyadh city, Saudi Arabia.

\section{CONCLUSION}

The pharmacist plays a significant role at critical care services of the private hospital. The pharmacist condition improved, decrease drug therapy cost and patient length hospital stay. Expanding critical care pharmacist at a private hospital is highly recommended and appreciated in the Kingdom of Saudi Arabia.

\section{ACKNOWLEDGMENT}

None.

\section{CONFLICT OF INTEREST}

The authors declare that there are no conflicts of interest.

\section{ABBREVIATIONS}

MOH: Ministry of Health; HMG: Dr. Sulaiman Al-Habib Medical Group; GCC: Gulf Cooperation Council.

\section{ORCID ID}

Yousef Ahmed Alomi, iD https://orcid.org/0000-0003-1381-628X

\section{REFERENCES}

1. Alomi YA, Alghamdi SJ, Alattyh RA. Strategic plan of general administration of pharmaceutical care at ministry of health in Saudi Arabia 2012-2022. J Pharm Pharm Scien. 2015;1(13):1-8.

2. Ahmed $Y$, Pharm A, Pharm C. National pharmacy practice programs at ministry of health in Saudi Arabia. Journal of Pharma and Pharmaceucal Sciences. 2015;1(2):17-8.

3. MacLaren R, Bond CA, Martin SJ, Fike D. Clinical and economic outcomes of involving pharmacists in the direct care of critically ill patients with infections. Crit Care Med. 2008;36(12):3184-9.

4. MacLaren R, Bond CA. Effects of pharmacist participation in intensive care units on clinical and economic outcomes of critically ill patients with thromboembolic or infarction-related events. Pharmacotherapy. 2009;29(7):761-8.

5. Leape LL, Cullen DJ, Clapp MD, Burdick E, Demonaco HJ, Erickson JI, et al. Pharmacist participation on physician rounds and adverse drug events in the intensive care unit. J Am Med Assoc. 1999;282(3):267-70.

6. The Society of Critical Care Medicine and the American College of Clinical Pharmacy. Pharmacotherapy. 2000;20(11):1400-6.

7. Schumock GT, Meek PD, Ploetz PA, Vermeulen LC. Economic evaluations of clinical pharmacy services 1988-1995. Pharmacotherapy. 1996;16(6):1188-208.

8. Alomi YA. Cost-efficiency of clinical pharmacy services at ministry of health in saudi arabia application of American Model. Value Heal. 2016;19(7):A482-3

9. Alomi YA, Almudaiheem HY, Alarnous T, Alshurei S, Alsharafa A, AlzahraniT, et al. Cost-efficiency of of national drug information center through ministry of healh hotline calling services (937) in Saudi Arabia: application of a Mercian Model. Value Heal J Int Soc Pharmacoeconomics Outcomes Res. 2015;18(7):A735.

10. Alomi YA, Alanazi AA, Alsallouk SA, Almaznai MM, Abu-Alnaja NI, Alduhilan M, et al. Cost-efficiency of medication safety program at pediatrics, obstetrics and gynecology hospital, East Province, Saudi Arabia. Value Heal. 2016;19(7):A464.

11. Alomi YA, Fallatah AO. Cost avoidance of pharmacist running pediatrics total parenteral nutrition services at ministry of health In Saudi Arabia. Value Heal. 2016;19(7):A461.

12. Aljbouri TM, Alkhawaldeh MS, Abu-Rumman AK, Hasan TA, Khattar HM, Abu-Oliem AS. Impact of clinical pharmacist on cost of drug therapy in the ICU. Saudi Pharm J. 2013;21(4):371-4.

13. Ling JM, Mike LA, Rubin J, Abraham P, Howe A, Patka J, et al. Documentation of pharmacist interventions in the emergency department. Am J Heal Pharm. 2005;62(17):1793-7.

14. Mutnick AH, Sterba KJ, Peroutka JA, Sloan NE, Beltz EA, Sorenson MK. Cost savings and avoidance from clinical interventions. Am J Heal Pharm. 1997;54(4):392-6

15. Alomi YA. National pharmacy practice programs at ministry of health in Saudi Arabia. J Pharm Pharm Scien. 2015;1(2):17-8.

16. Alomi YA. National drug information center program at Ministry of Health in Saudi Arabia. Adv Pharmacoepidemiol Drug Saf. 2016;5(1):1-2

17. Alomi YA. National medication safety program at ministry of health in Saudi Arabia. J Pharmacovigil. 2015;3(5):e145.

18. AlomiYA. National pharmacy pain management program at ministry of health in Saudi Arabia. J Pharmacol Clin Res. 2017;3(2):1-7.

19. Alomi YA. National antimicrobial stewardship program in Saudi Arabia; initiative and the future. J Surg. 2017;4(5):1-7. 